\title{
X-ray Polarization from Accreting Black Holes: Coronal Emission
}

\author{
Jeremy D. Schnittman \\ Department of Physics and Astronomy, Johns Hopkins University \\ Baltimore, MD 21218 \\ schnittm@pha.jhu.edu \\ and \\ Julian H. Krolik \\ Department of Physics and Astronomy, Johns Hopkins University \\ Baltimore, MD 21218 \\ jhk@pha.jhu.edu
}

\begin{abstract}
We present new calculations of X-ray polarization from accreting black holes (BHs), using a Monte-Carlo ray-tracing code in full general relativity. In our model, an optically thick disk in the $\mathrm{BH}$ equatorial plane produces thermal seed photons with polarization oriented parallel to the disk surface. These seed photons are then inverse-Compton scattered through a hot (but thermal) corona, producing a hard X-ray power-law spectrum. We consider three different models for the corona geometry: a wedge "sandwich" with aspect ratio $H / R$ and vertically-integrated optical depth $\tau_{0}$ constant throughout the disk; an inhomogeneous "clumpy" corona with a finite number of hot clouds distributed randomly above the disk within a wedge geometry; and a spherical corona of uniform density, centered on the $\mathrm{BH}$ and surrounded by a truncated thermal disk with inner radius $R_{\text {edge }}$. In all cases we find a characteristic transition from horizontal polarization at low energies to vertical polarization above the thermal peak; the vertical direction is defined as the projection of the $\mathrm{BH}$ spin axis on the plane of the sky. We show how the details of the spectropolarization signal can be used to distinguish between these models and infer various properties of the corona and BH. Although the bulk of this paper focuses on stellar-mass BHs, we also consider the effects of coronal scattering on the X-ray polarization signal from supermassive BHs in active galactic nuclei.
\end{abstract}

Subject headings: black hole physics - accretion disks - X-rays:binaries 


\section{INTRODUCTION}

A recent flurry of new mission proposals has renewed interest in measuring X-ray polarization from a variety of astrophysical sources. The Gravity and Extreme Magnetism SMEX $(G E M S)$ mission 1 , which has recently been approved for funding in the latest round of NASA Small Explorer proposals, should be able to detect a degree of polarization $\delta \lesssim 1 \%$ for a flux of a few mCrab (Black et al. 2003; Bellazzini et al. 2006; Swank et al. 2009). A similar detector for the International X-ray Observatory (IXO) could achieve sensitivity roughly $10 \times$ greater $(\lesssim 0.1 \%$ degree of polarization; Jahoda et al. 2007, Costa et al. 2008). Projects like these could potentially detect a large number of galactic and extra-galactic sources at the $\delta \sim 1 \%$ level, including stellar-mass black holes, magnetars, pulsar wind nebulae, and active galactic nuclei. In this paper, we focus on accreting black holes (BHs) in the "Hard" or "Steep Power Law" state (Remillard \& McClintock 2006), which are characterized by a broad-band spectrum with a thermal peak around $1 \mathrm{keV}$ and a strong high-energy powerlaw component extending above $100 \mathrm{keV}$. Early estimates suggest that the typical level of polarization from these sources should be a few percent in the $1-10 \mathrm{keV}$ range, depending on the geometry of the accretion system and the inclination to the observer (Connors et al. 1980; Sunyaev \& Titarchuk 1985). We also extend these results to AGN, whose hard X-ray properties are in many ways qualitatively similar.

Symmetry demands that in the Newtonian limit the observed polarization from a flat disk must be either parallel or perpendicular to its rotation axis. However, the effects of relativistic beaming, gravitational lensing, and gravito-magnetic frame-dragging can break that symmetry and give a non-trivial net rotation to the integrated polarization vector. Because the temperature in an accretion disk should increase closer to the $\mathrm{BH}$, where these relativistic effects are strongest, it was predicted long ago that the observed angle and degree of polarization of thermal disk emission should depend on photon energy (Stark \& Connors 1977; Connors \& Stark 1977; Connors et al. 1980). In more recent years, discussion of polarization in accreting black holes has expanded to include a number of other aspects of thermal disk emission, such as UV and X-ray emission from AGN disks (Laor et al. 1990; Matt et al. 1993) and "lamp post" models for irradiating the accretion disk with a non-thermal source above the plane (Dovciak et al. 2004). Quite recently, Dovciak et al. (2008) investigated the effect of atmospheric optical depth on the disk's polarization signal, and Li et al. (2008) applied the original calculations of thermal X-ray polarization to the problem of measuring the inclination of the inner accretion disk. Davis et al. (2009), using a Monte Carlo ray-tracing code on data from shearing-box simulations, estimated the effects of magnetic fields on the

\footnotetext{
${ }^{1}$ heasarc.gsfc.nasa.gov/docs/gems
} 
polarization of thermal emission. Schnittman \& Krolik (2009) (hereafter SK09) showed the importance of including returning radiation (Cunningham 1976) when calculating the polarization of BHs in the thermal state, an effect previously noted by Agol \& Krolik (2000).

In addition to the relatively well-understood thermal state, it has also long been known that most AGN and stellar-mass BHs can produce significant levels of harder X-rays with energies well above the thermal peak. In both cases, the hard flux is thought to be produced via inverse Compton scattering of the disk photons in a corona of hot (yet thermal) electrons (e.g. Haardt \& Maraschi (1993)). From the shape of this hard spectrum, the basic geometry and optical depth of the corona may be constrained (Haardt et al. 1994; Pietrini \& Krolik 1995; Stern et al. 1995; (Poutanen et al. 1997).

Despite these constraints, there remain sizable uncertainties about the nature and geometry of the hard X-ray emitting region. Popular models for the hard state of stellar-mass BHs include a cool disk truncated at large radius $(\sim 100 M)$ surrounding a hot, radiatively inefficient flow (Gierliński et al. 1997; McClintock et al. 2001; Esin et al. 2001; Done \& Trigo 2009), or alternatively a more extended disk surrounded by an optically thick hot corona, possibly in the form of a hot wind (Blandford \& Begelman 2004; Miller et al. 2006; Reis et al. 2009). Even less is known about the steep power-law (SPL) state. As for the hard state, most popular models are based on the inverse Compton scattering of thermal seed photons from a thin disk surrounded by a hot corona (Zdziarski \& Gierliński 2004), but bulk Comptonization of a converging accretion flow has also been suggested (Titarchuk \& Shrader 2002; Turolla et al. 2002). Although the geometry of the corona could be as simple as a uniform slab in the SPL state (Zdziarski et al. 2005), the hard state of galactic BH binaries, as well as the X-ray emission from AGN, are more likely caused by clumpy, inhomogeneous coronae, possibly caused by magnetic flares (Haardt et al. 1994; Poutanen \& Fabian 1999).

Here we explore the X-ray polarization signatures of three simple models for the corona geometry: a smooth sandwich with uniform optical depth in the vertical direction; an inhomogeneous model made of a finite number of spherical clouds, randomly distributed above the disk; and a truncated thin disk surrounding a spherical corona. In all cases, seed photons are emitted from the thermal, optically thick disk and are up-scattered in the hot corona (in the truncated disk case, we also include low-energy seed photons embedded in the corona). We find that in all cases, the spectrum comprises a thermal peak $(\sim 1-3 \mathrm{keV}$ for stellarmass BHs; $30-100 \mathrm{eV}$ for $\mathrm{AGN}$ ) and a power-law component dominating above that, which typically makes up $\sim 50-80 \%$ of the total flux. The gross features of the polarization spectra are quite robust: horizontal polarization with a few percent amplitude at low energies, with a transition to vertical orientation above the thermal peak, where the polarization amplitude can be as large as $\sim 10 \%$. The specific details of the polarization spectrum, i.e., 
the amplitude of polarization at low and high energies, and the shape and location of the transition, provide constraints on the global geometry, temperature, and optical depth of the corona, as well as the $\mathrm{BH}$ mass, spin, accretion rate, and the observer inclination angle.

In this paper, we begin in Section 2 with a brief overview of the computational methods used in the calculations. In Sections 35 we present our results for three different corona geometries: sandwich, hot spots/clumps, and a sphere embedded in a truncated disk, all in the context of galactic X-ray binaries. In Section 6, we apply the results to AGN. In Section 7 we discuss possible applications to observations.

\section{METHODOLOGY}

Although a detailed description of the ray-tracing code will be given in a companion paper (Schnittman \& Krolik 2010), we give a brief summary of the relevant physics here. The basic geometry is described by a thin disk with inner radius $R_{\text {edge }}$, typically placed at the inner-most stable circular orbit (ISCO), but possibly at smaller radii so long as $R_{\text {edge }}$ is outside the horizon. In the latter case, the gas inside the ISCO follows plunging trajectories along geodesics with energy and angular momentum determined by the ISCO values. The orbital angular momentum of the disk is prograde and aligned with the BH spin axis. Seed photons are emitted from the thin disk with a diluted black-body spectrum characterized by a hardening factor $f=1.8$ (Shimura \& Takahara 1995). The local flux is given by the Novikov-Thorne emissivity profile (Novikov \& Thorne 1973), but can be modified to include emission inside the ISCO, as described in SK09.

The seed photons have initial polarization parallel to the disk surface in the local fluid frame, as determined by the classical result for scattering-dominated atmospheres (Chandrasekhar 1960). The degree of polarization varies from zero for photons emitted normal to the disk surface up to $\sim 12 \%$ for an inclination angle of $90^{\circ}$. In addition to the polarization effects, the scattering of the outgoing flux causes limb-darkening, effectively focusing the emitted radiation in the direction normal to the disk surface. We define our seed photons in accordance with the polarization and limb-darkening factors tabulated as a function of emission angle in Table XXIV in Chandrasekhar (1960).

After leaving the disk, the photon packets follow geodesic trajectories around the black hole, eventually reaching a distant observer, returning to the disk via gravitational deflection, or getting captured by the horizon. En route, many photons scatter in the corona. We describe these events with the classical Thomson electron cross section. The differential 
probability for scattering along a path length $d l$ is simply

$$
P_{\text {scat }}=1-e^{-d \tau}=1-e^{-\kappa \rho d l},
$$

where $\kappa=0.4 \mathrm{~cm}^{2} \mathrm{~g}^{-1}$ is the opacity to electron scattering, $\rho$ is the local mass density of the corona, and $\tau$ is the optical depth. Unlike some recent Monte Carlo ray-tracing codes (e.g. Davis et al. (2009); Dolence et al. (2009)) that use the more physically accurate, energydependent Klein-Nishina cross section, the Thomson cross section allows for the use of photon packets that include the entire broad-band spectrum, as described in Schnittman \& Krolik (2010). This simplification gives improved computational efficiency, albeit at the loss of physical accuracy for above $\sim 200 \mathrm{keV}$, where the classical and relativistic electron cross sections begin to diverge. For most stellar-mass BH sources, and for most X-ray polarimetry missions in the foreseeable future, it should be quite safe to focus on photons with energies less than $100 \mathrm{keV}$.

At each step along the photon geodesic, the probability for scattering is calculated according to equation (1), and then a uniform random number in $[0,1)$ determines whether that photon scatters at that location 2. When the photon does scatter, we first transform the photon 4-momentum $\mathbf{k}$ and polarization vector $\mathbf{f}$ into the local inertial frame of the corona, then do a special-relativistic boost into the electron's rest frame, where the electron velocity is taken from an isotropic thermal distribution. In the electron's rest frame, the scattering event is treated completely classically, with a differential cross section given by (Rybicki \& Lightman 1979)

$$
\frac{d \sigma}{d \Omega}=\frac{1}{2} r_{0}^{2}\left[(1-\delta)\left(\cos ^{2} \Theta+1\right)+2 \delta \cos ^{2} \Theta \cos ^{2} \psi+2 \delta \sin ^{2} \psi\right],
$$

where $r_{0}=2.82 \times 10^{-13} \mathrm{~cm}$ is the classical electron radius, $\delta$ is the degree of polarization, $\Theta$ is the angle between incident and outgoing photon directions, and $\psi$ is the polarization angle, measured with respect to the scattering plane. The angles $\Theta$ and $\psi$ are selected appropriately from the probability distribution corresponding to (2). The outgoing Stokes parameters are given by

$$
\begin{aligned}
I^{\prime} & =\frac{3}{2}\left(I_{\|} \cos ^{2} \Theta+I_{\perp}\right) \\
Q^{\prime} & =\frac{3}{2}\left(I_{\|} \cos ^{2} \Theta-I_{\perp}\right) \\
U^{\prime} & =\frac{3}{2} U \cos \Theta
\end{aligned}
$$

\footnotetext{
${ }^{2}$ Our adaptive-step Cash-Karp geodesic integrator takes smaller steps where the electron density is high, ensuring that $d \tau<<1$.
} 
where $I_{\|}$and $I_{\perp}$ are the components of the intensity with polarization parallel to and perpendicular to the scattering plane, respectively. These Stokes parameters are then used to reconstruct the new polarization degree and angle through

$$
\begin{aligned}
& X=Q / I, \\
& Y=U / I
\end{aligned}
$$

and

$$
\begin{aligned}
\delta & =\left(X^{2}+Y^{2}\right)^{1 / 2}, \\
\psi & =\frac{1}{2} \tan ^{-1}(Y / X) .
\end{aligned}
$$

The scattered photon packet is boosted back to the coronal fluid frame, then transformed back into the coordinate basis and continues propagating along its new geodesic. This "transform-boost-scatter-boost-transform" algorithm automatically carries out the inverse Compton change in photon energy, giving the outgoing photon an average energy increase of $\gamma^{2}$, where $\gamma$ is the electron Lorentz factor $\left(\gamma \approx 1+k T /\left(m_{e} c^{2}\right)\right.$ for thermal electrons $)$. The polarization 4 -vector is parallel-transported simply by satisfying the constraints $\mathbf{k}$. $\mathbf{f}=0$ and $\mathbf{f} \cdot \mathbf{f}=1$ and using the complex-valued Walker-Penrose integral of motion $\kappa_{\mathrm{WP}}$ (Walker \& Penrose 1970). Analogous to the way that Carter's constant (Carter 1968) can be used to constrain 4 -velocity components, $\kappa_{\mathrm{WP}}$ can be used to reconstruct the polarization vector at any point along the geodesic. The two orthonormality conditions stated above, along with the real and imaginary parts of $\kappa_{\mathrm{WP}}$, give a total of four equations for the four components of the polarization vector $\mathbf{f}$.

Some photons encounter the disk before reaching their final destination. When we treat models relevant to AGN, photons striking the disk are absorbed because our primary interest is in photons $<10 \mathrm{keV}$, and AGN disks are generally thought to have enough photo-ionization opacity to have little albedo at these energies. In the stellar-mass black hole case, disks are expected to be highly reflective. For these models, we use the results given in Section 70.3 of Chandrasekhar (1960) for diffuse reflection, i.e. multiple scattering events in a semi-infinite plane.

When a ray reaches an observer at infinity, the polarization vector is projected onto the detector plane. In our convention, the y-axis of the detector is parallel to the projected symmetry axis of the black hole and accretion disk, so $\psi=0^{\circ}$ corresponds to "horizontal" polarization and $\psi= \pm 90^{\circ}$ is "vertical." Finally, we integrate over all photon bundles to obtain the energy-dependent Stokes parameters $I_{\nu}, Q_{\nu}$, and $U_{\nu}$, which in turn give $\delta_{\nu}$ and $\psi_{\nu}$, our preferred observables for most of the results presented below. 


\section{WEDGE GEOMETRY}

We begin by considering the simplest corona geometry, an isothermal layer of uniform vertical optical depth forming a wedge with constant opening angle $\tan \theta_{c}=H / R$. At each point in the disk, the atmosphere has an exponential profile in the vertical direction with $\rho(z, R)=\rho_{0} \exp [-z / H(R)]$. The vertically-integrated optical depth $\tau_{0}$ is constant, giving

$$
\rho_{0}(R)=\frac{\tau_{0}}{\kappa H(R)} .
$$

We set the local rest frame of the corona to be corotating with the underlying disk: $u^{r}=$ $u^{\theta}=0, u^{\phi} / u^{t}=\Omega(R)$, and $u^{\mu} u_{\mu}=-1 . \Omega(R)$ is the orbital frequency (measured at infinity) of a planar circular orbit at radius $R$, which in Boyer-Lindquist coordinates is

$$
\Omega(R)=\frac{1}{(R / M)^{3 / 2}+a / M} .
$$

Figure1gives a schematic view of the accretion geometry, showing how the seed photons originate in the midplane, then scatter through the hot corona before reaching a distant observer. The coronal scattering has two major effects on the observed polarization signature. First, it changes the underlying spectrum by inverse Compton scattering a portion of the photons, boosting them to higher energies, and thus producing a harder spectrum. Second, it changes the amplitude and orientation of the net polarization at high energies, rotating it from horizontal to vertical, similar to the way returning radiation gets scattered into a vertical orientation by a thermal disk (SK09). The coronal polarization rotation effect is essentially the same as that described in Sunvaev \& Titarchuk (1985), who considered the up-scattering of low-energy photons embedded in a hot electron corona with planar geometry, finding strong vertical polarization for high-energy photons and observers at high inclinations.

The cause of this rotation in systems with moderate optical depth $\left(\tau_{0} \lesssim 2\right)$ can be understood easily from the scattering geometry in Figure 1. Photons initially emitted in directions near the disk plane are almost certain to scatter because they face a large optical depth. To reach an observer who views the disk nearly edge-on, they must stay in the disk plane even after scattering, but this requires a vertical polarization direction. Additionally, those photons that scatter multiple times in the corona-and are thus boosted to higher energies - are geometrically more likely to move in the plane of the corona, parallel to the disk. This further increases the amplitude of their vertical polarization and preferentially scatters them to infinity with large emission angle, leading to a limb-brightening effect at high energies with respect to the classical Chandrasekhar result (Sunyaev \& Titarchuk 1985). 
For hot coronae with high optical depth, the multiple scatters cause the polarization and limb-darkening to tend towards the Chandrasekhar limit, but with the photon energy dependence given by a Wien spectrum. In the limit of a cold corona, where there is no transfer of energy via inverse Compton scattering, there is no way to distinguish between photons coming directly from the disk atmosphere and those that are scattered in the corona, and the classical result is reproduced regardless of the coronal optical depth.

To improve our qualitative understanding of the polarization effects of the global corona geometry, we plot in Figure 2 a series of images of the $\mathrm{BH}$ accretion disk plus corona, sorted by the scattering history of each photon. Figure 2 a shows only photons that travel directly from the disk to the observer; Figure 2 b shows flux from return radiation - photons that are bent by gravitational lensing and scatter off the disk to the observer; Figure 2 c is the flux from photons that scatter exactly once in the corona; and Figure $2 \mathrm{~d}$ is made of photons that scatter multiple times in the corona. The observed intensity is color-coded on a logarithmic scale (normalized to the peak intensity from all photons) and the energy-integrated polarization vectors are projected onto the image plane with lengths proportional to the local degree of polarization. The black hole has a spin of $a / M=0.9$, mass $M=10 M_{\odot}$, and accretion rate such that the thermal flux alone totals $10 \%$ of the Eddington luminosity. The corona has a temperature of $100 \mathrm{keV}$, scale height $H / R=0.1$, and vertical optical depth $\tau_{0}=1$. These parameters lead to a coronal luminosity $2-3$ times that of the thermal luminosity. The disk is rotating in the counter-clockwise direction, and the observer is located at an inclination of $75^{\circ}$ to the rotation axis. The intensity maximum at the left of each image is caused by relativistic beaming and Doppler boosting. Photons from the far side of the disk are bent by gravitational lensing, giving the appearance of a warped disk with the far side having a smaller effective inclination angle.

Not surprisingly, the direct image looks quite similar to that for the direct radiation from a simple thermal disk with no corona (SK09). The polarization is modest and predominantly horizontal, with relativistic effects most noticeable in the inner disk, where beaming and lensing combine to rotate the observed angle of polarization at higher energies. The degree of polarization is reduced above and to the left of center, where the effective inclination of the disk is smaller, while the opposite occurs in the receding section of the disk on the right side of the image. However, when carefully compared to the non-corona system (Fig. 1 of SK09), we see a clockwise shift in the peak flux distribution because the level of direct flux from a given patch of the disk is dependent on the optical depth to scattering along the geodesic connecting that patch with the observer. For the wedge geometry, this means that where the effective inclination is smallest, the observed flux is greatest, leading to additional limb-darkening in the direct component. 
The return radiation image is also similar to that for the thermal disk alone, although now the presence of a scattering corona strongly suppresses any return flux from large radii. Since most returning photons pass very close to the light orbit around $R=3 M$, they would necessarily intersect the outer disk at very high angles of incidence, and thus face very large optical depth through the wedge corona. As shown in SK09, the flux from returning radiation, while small compared to the total flux, is very highly polarized, and in a vertical direction, perpendicular to that of the direct flux. Since the majority of the returning radiation originates in the hot inner regions of the disk, there is a dependence of the polarization direction on photon energy. As can be seen from Figure $2 b$, the angle of polarization for the return radiation is negative, as measured in the image plane, with $\psi=0$ defined along the $x$-axis. This can also be understood from the global geometry of the system, as shown in Figure 1. Photons emitted from the right side of the disk, deflected by the BH and incident on the left side at some finite angle, will scatter towards a high-inclination observer with large polarization in the $\psi<0$ orientation. Of course, the opposite occurs for photons emitted from the left side and scattered on the right side, but they get preferentially scattered away from the observer by relativistic beaming.

In Figure 2k we show the flux from photons experiencing a single coronal scatter. Since every photon in this image scatters exactly once, there is no preference for oblique emission, and photons emitted at all inclination angles are equally weighted. If anything, for $\tau_{0}=1$, the seed photons emitted normal to the disk surface are more likely to scatter exactly once and then escape to an observer at high inclination, thus explaining the prevailing horizontal polarization seen here. Lastly, Figure $2 \mathrm{~d}$ shows the multiply-scattered photons. These have strong net vertical polarization due to the geometric effects described above and shown schematically in Figure 1,

Unlike the returning radiation, the coronal flux has a net positive polarization angle $\psi>0$ when viewed edge-on. This can again be understood from the schematic image of the wedge corona in Figure 1. It is clear from this geometry that for two photons, both emitted in the plane of the sky at the same angle $i_{\mathrm{em}}$ to the disk normal, the photon emitted in the direction away from the black hole will have a greater path length within the wedge corona. However, due to the increasing scale height and constant optical depth in the vertical direction, it will pass through lower-density gas. Combining these two effects - path length and coronal density as a function of emission angle - we find that the optical depths for ingoing and outgoing photons are

$$
\tau_{\mathrm{in}}\left(i_{\mathrm{em}}\right)=\frac{\tau_{0}}{2}\left[\frac{\cos \theta_{c}}{\cos \left(i_{\mathrm{em}}-\theta_{c}\right)}+\frac{1}{\cos i_{\mathrm{em}}}\right]
$$


and

$$
\tau_{\text {out }}\left(i_{\mathrm{em}}\right)=\frac{\tau_{0}}{2}\left[\frac{\cos \theta_{c}}{\cos \left(i_{\mathrm{em}}+\theta_{c}\right)}+\frac{1}{\cos i_{\mathrm{em}}}\right] .
$$

Here $\theta_{c}$ is the opening angle of the corona and we restrict $i_{\mathrm{em}}<\pi / 2-\theta_{c}$ so that the outgoing optical depth is finite.

Since $\tau_{\text {out }}>\tau_{\text {in }}$ for each $i_{\mathrm{em}}$, the "tilt" of the polarization vector for corona scattering is opposite that of return radiation. For the region of the disk beamed towards the observer (left side of images in Fig. 2), this means that photons emitted in the direction away from the $\mathrm{BH}$ are more likely to be scattered in the wedge corona, giving a net polarization oriented with $\psi>0$, as can be seen in the lower panels of Figure 2. In practice, we can't ever really know whether a given photon came directly from the disk or was scattered in the corona or off the disk, but the images do provide important qualitative understanding of the geometric effects involved.

For a more quantitative picture, we plot in Figure 3 the observed flux, polarization degree, and polarization angle as a function of energy for observer inclinations of $i=45^{\circ}, 60^{\circ}$, and $75^{\circ}$. We sort the photons into direct (dotted curves), scattered (dot-dashed curves), and total flux (solid curves). For a $\mathrm{BH}$ mass of $10 M_{\odot}$ accreting at a rate $\sim 0.1 L_{\mathrm{Edd}}$, the thermal peak is near $1 \mathrm{keV}$, with essentially no flux from the disk above $\sim 10 \mathrm{keV}$. With $\tau_{0}=1$ and $T_{c}=100 \mathrm{keV}$, the Compton $y$ parameter is of order unity, so that the up-scattered spectrum has $F_{\nu} \propto \nu^{-1}$ (Rybicki \& Lightman 1979). As the inclination of the observer increases, the optical depth to the disk increases, thus lowering the relative contribution from the direct radiation. In all cases, the up-scattered radiation dominates the flux above a few keV.

The direct radiation behaves quite similarly to that described in Connors et al. (1980), reproducing the Chandrasekhar result at low energy, and then decreasing in fractional polarization at higher energies as the relativistic effects of the inner disk begin to dominate. As discussed in SK09, the angular rotation in $\psi$ is greatest for small observer inclinations simply because there is a smaller degree of "classical" polarization to overcome.

When considering only the scattered photons, the polarization is still given by the Chandrasekhar limit at low energies. The scattered photons below the thermal peak have typically scattered only once in the corona, thus preserving their initial horizontal orientation (see Fig. 2k). At higher energies, we begin to sample the multiply-scattered photons that make up Figure 2 d, giving strong vertical polarization. Thus there is a transition from horizontal to vertical orientation even when considering the scattered photons alone. When including all the flux (solid curves), this transition point is shifted to somewhat higher energies than for the scattered photons alone due to the horizontal contribution of the direct thermal radiation. 
As mentioned above, the effects of return radiation and coronal scattering on the sign of the polarization angle are opposite: return radiation leads to a negative angle and the coronal scattering in a wedge geometry leads to a positive angle in the transition from horizontal to vertical polarization. However, some photons that scatter in the corona are also deflected by gravitational lensing and then scattered in the corona on the far side of the disk, leading to some ambiguity as to whether they should be classified as return photons or coronal photons. From Figure 3 we see that the relative contributions from these two effects are also dependent on the observer inclination, with low-inclination systems giving a negative tilt and high-inclination leading to a positive tilt. This distinction may be observable in practice by comparing the polarization in the hard state to that of the soft state, where the transition direction is independent of inclination and therefore can be used to define the negative orientation (SK09).

Using spectropolarimetry to infer physical properties of the source is certainly more complicated in the hard state than in the thermal state, for the simple reason that there are more free parameters in the underlying model, creating degeneracies in fitting the data. Instead of attempting to quantify our ability to measure any particular property of the $\mathrm{BH}$ system, we present here the effects of separately changing individual parameters with respect to a fiducial model with $M=10 M_{\odot}, a / M=0.9, L_{\text {therm }}=0.1 L_{\text {Edd }}, i=75^{\circ}, \tau_{0}=1.0$, $T_{c}=100 \mathrm{keV}$, and $H / R=0.1$.

In Figure 4 we show the polarization degree and angle for the fiducial model, varying the luminosity in the thermal flux component from $0.01 L_{\text {Edd }}$ to $L_{\text {Edd }}$. Since in all cases the thermal peak is well below the corona temperature, the transition energy scales linearly with the disk temperature, which is in turn proportional to the quarter power of the luminosity. With multiple observations of a single source accreting at various rates as in Figure 4, one could confirm that the thermal part of the spectrum is indeed coming from a thin disk with constant $R_{\text {edge }}$ Gierliński \& Done 2005).

Holding the net thermal flux fixed, but varying the spin, changes the emissivity profile of the disk, concentrating more of the emission close to the BH for larger spins (and thus smaller $\left.R_{\mathrm{ISCO}}\right)$. As in the thermal state (SK09), this leads to a greater fraction of return radiation, and thus the transition from horizontal to vertical polarization occurs at lower energy, as shown in Figure 5. In the hard/SPL states, the presence of a corona provides another, complementary mechanism for effecting the polarization transition above the thermal peak, regardless of the spin parameter. Thus the dependence of the polarization signal on BH spin is weaker than in the thermal state. In this way, the sandwich corona acts as a veil around the inner disk, obstructing our view of the plunging region where the orbital dynamics are most sensitive to the spin. 
In Figure 6, we simultaneously vary the coronal optical depth and temperature, maintaining a roughly constant Compton $y$-parameter. In the wedge corona model, the transition from horizontal to vertical polarization is largely due to local geometric effects (as opposed to more global effects like returning radiation). In this case, the optical depth and opening angle of the corona largely determine the scattering history of each photon, and thus the polarization degree and angle of the outgoing radiation. Holding the size and shape of the corona constant, two photon packets experiencing the same number of scattering events will emerge on average with the same polarization signature. Thus the energy of the transition is primarily a function of the scattering electron temperature, and hotter coronae lead to a higher-energy transition. Similarly, to reach a given energy above the thermal peak, photons have to scatter more times in a cooler corona, resulting in a more constrained scattering history, and thus higher polarization. At the same time, when changing the optical depth of the corona, the overall scattering geometry does change slightly, so the shape of the transition also varies in Figure 6. Furthermore, for small optical depths, a larger fraction of the photons can return to the disk, giving the negative polarization angle characteristic of returning radiation.

Lastly, we show in Figure 7 the effect of varying the scale height of the corona. Here, the fact that return radiation and coronal scattering rotate the polarization angle in opposite senses away from horizontal leads to a complicated dependence. The geometrically thinnest coronae behave similarly to the razor-thin thermal disks described in SK09, giving a more gradual transition from horizontal to vertical with $\psi<\sqrt{3}$. As the opening angle of the corona increases, the transition flips to $\psi>0$ as the coronal scattering effects of equation (8) becomes more important. For very large $H / R$, the lower density of the corona allows longer path lengths and photons can sample a larger volume of the accretion flow. At low energies this loosening of the geometrical constraints leads to a deviation from the classical planar scattering atmosphere. At higher energies, it leads to a higher effective temperature as the bulk velocity of the corona (which can be a substantial fraction of $c$ ) is added to the thermal velocities of the scattering electrons. The combination of these various effects eliminates the existence of any simple trends in Figure 7.

\footnotetext{
${ }^{3}$ From eqn. (8) we see that when $\theta_{c} \rightarrow 0$, there is no difference between photons emitted towards or away from the $\mathrm{BH}$, leaving only the global effects of returning radiation.
} 


\section{INHOMOGENEOUS GEOMETRY}

Motivated by spectral models of AGN Stern et al. (1995), whose reasoning also applies to galactic BHs in the hard state Poutanen et al. (1997), we also consider an inhomogeneous geometry where the hot coronal plasma is clumped into a large number of small, dense regions. These clouds are distributed randomly above the disk, roughly following a wedge geometry. For precisely the same reasons that originally motivated the clumpy corona model, it is not generally possible to reproduce the same spectrum as in the sandwich geometry. Yet we find that by fixing the electron temperature and conserving the total mass in the corona, the net flux in hard X-rays $(\gtrsim 10 \mathrm{keV})$ is roughly the same.

We describe the inhomogeneous corona with five parameters: aspect ratio $H / R$, temperature $T_{c}$, mean vertical optical depth $\tau_{0}$, number of clouds per unit radius $2 n_{c}\left(n_{c}\right.$ above the plane and $n_{c}$ below the plane; $n_{c} \equiv d N_{c} / d R$ is constant throughout the disk, giving a larger number of clouds per unit area in the inner disk), and an overdensity factor $\rho_{c} / \rho_{0}$, where $\rho_{0}(R)$ is the mean density of a wedge corona with the same scale height and optical depth. The distribution of clouds is chosen so as to reproduce the wedge corona in the limit of $n_{c} \rightarrow \infty$ and $\rho_{c} / \rho_{0} \rightarrow 1$. To do this, we require that the average mass in the inhomogeneous corona is equal to that of a wedge corona at each annulus $(R, R+d R)$. For spherical clouds of radius $R_{c}(R)$, the coronal mass above (or below) the disk at each annulus is given by:

$$
2 \pi \rho_{0} H R d R=n_{c} \frac{4}{3} \pi \rho_{c} R_{c}^{3} d R
$$

which gives a clump radius

$$
R_{c}(R)=\left[\frac{3}{2}\left(\frac{H}{R}\right) \frac{R^{2}}{n_{c}\left(\rho_{c} / \rho_{0}\right)}\right]^{1 / 3} .
$$

The covering fraction can be estimated by calculating the probability that a given region of the disk is not covered by a coronal cloud. Consider an annulus of area $2 \pi R \Delta R$. The expectation value for the number of clouds over it is $N=n_{c} \Delta R$. If each cloud has cross sectional area $\pi R_{c}^{2} \ll 2 \pi R \Delta R$, this probability is given by

$$
p=\left(1-\frac{\pi R_{c}^{2}}{2 \pi R \Delta R}\right)^{N} \underset{N \rightarrow \infty}{\rightarrow} \exp \left(-\frac{n_{c} R_{c}^{2}}{2 R}\right)=\exp \left[-\frac{1}{2} n_{c}^{1 / 3} R^{1 / 3}\left(\frac{3}{2} \frac{H}{R} \frac{\rho_{0}}{\rho_{c}}\right)^{2 / 3}\right] .
$$

The covering fraction is simply $f_{c}=1-p$.

In Figure 8 we show a series of images of the $\mathrm{BH}$ accretion disk plus a clumpy corona, again sorted by photon history as in Figure 2. The corona is made up of 100 clouds distributed randomly with scale height ratio $H / R=0.1$ with constant probability per unit 
radius (i.e., $d N / d R=n_{c}=1$ ) inside radius $R=100 M$. The mean local optical depth $\tau_{0}=1$, and the density contrast $\rho_{c} / \rho_{0}=10$. For the clumping algorithm described above, these parameters correspond to a covering fraction in the inner disk of $f_{c} \sim 5 \%$, comparable to that inferred for the hard state of Cyg X-1 by Poutanen et al. (1997). As in Figure 2, the $\mathrm{BH}$ has spin $a / M=0.9$, mass $10 M_{\odot}$, thermal flux $L_{\text {therm }}=0.1 L_{\mathrm{Edd}}$, corona temperature $T_{c}=100 \mathrm{keV}$, and observer inclination angle $75^{\circ}$. The direct flux from the disk, plotted in Figure $8 \mathrm{a}$, clearly shows a number of distinct shadows where optically thick clouds block the disk.

The reduced covering fraction of the corona leads to a greater flux from return radiation (Fig. 8b), which otherwise behaves much the same as in the sandwich geometry, contributing a strong vertical component to the polarization signal. As in the direct image, the return radiation that eventually reaches the observer is also blocked in places by intervening coronal patches. In the lower panels of Figure 8, we show the scattered photons (Fig. 86: one scatter; Fig. 8f: many scatters). Now the polarization of the hard flux is significantly less coherent than in the sandwich geometry. Many photons scatter multiple times within a single cloud, eventually emerging with no particular polarization direction. At the same time, the global geometry of the system is still essentially the same as the sandwich model, and photons are more likely to experience multiple scattering events by propagating from one coronal clump to another, constrained to move roughly parallel to the disk surface. As in the homogeneous model, this leads to a net vertical polarization in the observed flux at high energies.

In Figure 9 we plot the flux, polarization degree, and polarization angle as a function of energy for the inhomogeneous corona and observer angles of $45^{\circ}, 60^{\circ}$, and $75^{\circ}$. As in the sandwich geometry, here too we see that with increasing inclination, a greater fraction of the observed flux comes from scattered photons. Due to the smaller covering factor, a greater fraction of the thermal flux can escape at all inclinations, but the spectrum is still dominated by Comptonized photons above $7-8 \mathrm{keV}$. From equation (10), we see that the individual coronal clumps have typical optical depths of $\tau \approx 10$ in the inner disk. This naturally leads to a harder power-law spectrum relative to that of the wedge geometry.

The polarization signature for the inhomogeneous corona is similar to the sandwich geometry at low energies, but noticeably different above a few keV. Below the thermal peak, a sandwich corona merely extends the classic Chandrasekhar atmosphere, leaving the polarization unchanged. The scattering contribution (dot-dashed curves) is more weakly polarized when the gas is clumped because it is easier for a photon emitted at a large angle relative to the disk normal to scatter once off a coronal cloud and then reach an observen, contributing

\footnotetext{
${ }^{4}$ The clumpy corona can be thought of as a collection of hard scattering spheres where photons are unable
} 
a vertical component to the polarization, slightly diluting the dominant horizontal signal at low energy. On the other hand, the degree of polarization at high energies is significantly lower for a clumpy corona than for the sandwich corona. This is because many high-energy photons scatter multiple times within a single cloud, ultimately emerging with little or no net polarization due to the spherical symmetry of the cloud.

Lastly, we note that when the corona is clumped, the shape of the transition from horizontal to vertical polarization more closely resembles that of the thermal state than that of a homogeneous sandwich corona: relatively smooth and in the $\psi<0$ direction. Because of the small covering factor of the corona, thermal seeds emitted in the inner disk have a greater chance of returning to the disk or even scattering off a coronal clump on the far side of the $\mathrm{BH}$, thus leading to a $\psi(E)$ distribution quite similar to that of the thermal disk with no corona (SK09). Not surprisingly, the polarization signature is sensitive to the compactness of the coronal hot spots, as shown in Figure 10. In the limit of $\rho_{c} / \rho_{0}=1$, the signal more closely resembles the homogeneous sandwich result: a sharp transition at $2-3 \mathrm{keV}$, with $\psi>0$ and the polarization degree rising above $6 \%$ at high energies (this case does not identically reproduce the homogeneous sandwich when $N$ is finite because, although the centers of the clouds must lie within $H$ of the plane, their outer portions may extend farther, resulting in an inhomogeneous geometry even when $\rho_{c} / \rho_{0}=1$ ). As the compactness increases, the covering fraction and the overall symmetry decrease, giving a smoother transition and smaller amplitude above a few keV. In Figure 11 we show the dependence of the polarization signal on the number of clumps in the corona, holding the overdensity fixed at $\rho_{c} / \rho_{0}=10$. In the limit of $N_{\text {clump }} \rightarrow \infty$, the homogeneous result is reproduced, and in the limit of $N_{\text {clump }} \rightarrow 0$ we get the pure thermal result.

\section{SPHERE GEOMETRY}

The last model we consider is a spherical corona immediately surrounded by a truncated disk, a geometry motivated by a variety of spectral and timing observations (e.g. Gierliński et al. (1997); Done \& Zycki (1999); Makishima et al. (2008); Ingram et al. (2009); Done \& Trigo (2009)). The disk extends in to radius $R_{\text {edge }}$ with a Novikov-Thorne emissivity profile, and the corona is defined by its temperature $T_{c}$ and optical depth $\tau_{0}$, as measured from the horizon out to $R_{\text {edge }}$. The electron density in the corona is taken to be constant, and in keeping with its spherical shape, the fluid is at rest in the frame of a zero-angular momentum observer (Bardeen et al. 1972). In order to most closely match the spectral properties of

to forward-scatter, unlike the homogeneous atmosphere of diffuse electrons. 
the sandwich corona, we also embed thermal seed photons with a uniform distribution inside the corona. The net flux and spectrum as a function of radius is determined by matching the shell-integrated flux to that of a standard Novikov-Thorne disk down to the ISCO. These coronal seed photons are emitted isotropically with zero polarization.

In Figure 12 we show the same photon-sorted images as in Figure 2, now for the spherical coronal model with $R_{\text {edge }}=15 \mathrm{M}$. The direct disk flux behaves as expected: because it is emitted from regions that are almost entirely in the non-relativistic regime, its polarization is very close to the Chandrasekhar value. There is now also a significant amount of unpolarized thermal flux coming directly from the coronal seeds. These seed photons can also scatter off the surrounding disk, in which case they are classified here as return radiation, again strongly polarized in the vertical direction. The corona-scattered flux shown in Figures $12 \mathrm{r}$, d closely resembles that of a scattering-dominated atmosphere around a star or planet: zero polarization from surfaces normal to the observer, and strongly-polarized (but limbdarkened) flux from the edges. However, the disk blocks a portion of the bottom half of the sphere, with the very bottom the most likely to be blocked. Consequently, the net polarization from a spherical corona is non-zero and oriented in the vertical direction. We have not shown here the contribution from photons that scatter in the corona and then off the disk, but these also are strongly polarized in the vertical direction, much like the image in the upper-right panel.

Just as in the analogous figures for the sandwich and clumped models, Figure 13 shows that the total flux spectrum is dominated at low energies by the thermal contribution (here the sum of the disk proper and the thermal seeds embedded in the corona), while it is dominated at high energies by inverse Compton scattered photons. Because the coronal density and temperature were specifically chosen to give the same Compton $y$-parameter as in the sandwich geometry (see Fig. 31), it is not surprising that the spectra agree so closely.

The polarization signature is relatively straightforward to understand, especially in the context of Figure 12: the polarization from the direct radiation is almost exactly horizontal, decreasing steadily in amplitude with energy as a greater proportion of the observed flux comes from the unpolarized and higher-energy seeds in the corona. The scattered flux is consistently vertical, since even the lower-energy photons that have scattered only once have a net vertical orientation (see the lower-left panel of Fig. 12). Because the unobscured coronal hemisphere looks much the same from each of the three viewing angles, the polarization amplitude at high energies is only weakly dependent on observer inclination. Combining these effects gives the now-familiar behavior of horizontal polarization at low energies governed by the Chandrasekhar limit transitioning to vertical orientation above the thermal peak. The spherical geometry leads to a particularly sharp transition, at least for $R_{\text {edge }} \gtrsim 10 M$, 
but it is still clear that a negative $\psi$ is preferred in the transition region. Again this can be understood by the geometry of the return radiation scattering off the region of the disk most strongly beamed towards the observer. In this case, the coronal photons originating from the central region above the disk plane replace the return radiation that similarly had passed near the photon orbit, just above the $\mathrm{BH}$.

In Figure 14 we investigate the effects of varying $R_{\text {edge }}$, while keeping the total optical depth of the corona constant. Since the thermal disk moves in with decreasing $R_{\text {edge }}$, we find the transition point moves to higher energies, as a greater fraction of the flux is coming directly from the disk and is therefore horizontally polarized. The shape of the transition grows broader with decreasing $R_{\text {edge }}$ as relativistic effects become more important, rotating the polarization angle as in the thermal state. In the limit of large $R_{\text {edge }}$, where Newtonian physics dominates the problem, the system becomes scale-invariant and symmetry constrains the polarization at any given energy to be exactly horizontal or exactly vertical. In the limit of very small $R_{\text {edge }}$, we reproduce the thermal result, plus a small power-law contribution to the spectrum at high energy due to the ultra-compact corona.

Holding the corona radius constant at $R_{\text {edge }}=10 M$, in Figure 15 we show the effects of varying the optical depth and temperature of the corona. As in Figure 6 for the sandwich geometry, here too we keep the Compton $y$-parameter fixed so that the resulting spectra are nearly identical. However, unlike the sandwich model, for the spherical corona we find essentially no dependence on the coronal temperature or density. The reason is the essential simplicity of the scattering geometry: the polarization signature from the direct radiation alone is nearly the same, regardless of the coronal optical depth, and from Figure 13 we see that all scattered radiation is polarized exactly the same over the entire spectrum. Thus the behavior of the net signal is simply a function of the relative flux in each component, and this is held constant by fixing the Compton $y$-parameter.

\section{ACTIVE GALACTIC NUCLEI}

It has long been known that a significant fraction of the flux from AGN is emitted in the X-ray band (Elvis et al. 1978). As in stellar-mass BHs, this high-energy flux likely comes from lower-energy seed photons inverse Compton scattered in a corona of hot electrons with $T_{c} \sim 100 \mathrm{keV}$. Similar to the stellar-mass case, this leads to a relatively hard power-law spectrum with index $\alpha \sim 0.5-1$ (Nandra et al. 1991; Mushotzky et al. 1993). However, unlike the stellar-mass case, the temperature of the inner disk for an AGN will be well below a keV, leading to a thermal peak in the UV band. Furthermore, even when the disk is dominated by radiation pressure and electron scattering opacity, there should still 
be a substantial fraction of metals that are not fully ionized, producing a large opacity for absorption above $\sim 1 \mathrm{keV}$.

Both of these AGN features - lower energy seed photons and an X-ray absorbing disklead to important differences in the polarization signature as compared to the stellar-mass models described above in Sections [35. Because the seed photons start off with lower energies, they must scatter more times in the corona in order to reach the $\sim 1-10 \mathrm{keV}$ band. For a thin sandwich corona, this means that the scattering geometry is even more constrained than in the stellar-mass case, forcing the photons to move in a plane parallel to the disk surface, leading to a stronger vertical polarization. The AGN disk absorbs much of the incident X-ray flux from the corona, so the Compton $y$-parameter is effectively smaller than that of a stellar-mass system with the same coronal properties because scattering sequences are halted once a photon strikes the disk (we do not re-radiate the X-ray flux, but rather treat it as completely absorbed), thereby reducing the average path length the photons that escapes to infinity. An absorbing disk boundary condition with a sandwich corona therefore leads to an even higher degree of X-ray polarization because the photons are forced to scatter in a more constrained geometry before escaping the corona.

In Figure 16 we show broad-band spectra from an AGN with central mass $M=10^{7} M_{\odot}$, spin parameter $a / M=0.9$, thermal luminosity $L_{\text {therm }}=0.1 L_{\text {Edd }}$, and observer inclination angle $i=45^{\circ}$. Four different corona models are considered: a homogeneous wedge with $H / R=0.1, \tau_{0}=1$, and $T_{c}=100 \mathrm{keV}$, and three clumpy models with the same scale height, mean optical depth, and temperature, but with $n_{c}=1$ and overdensities of $\rho_{c} / \rho_{0}=1$, 3 , and 10. The inhomogeneous models can be characterized by their covering fractions as measured in the inner disk at $R=10 \mathrm{M}$; these overdensities correspond to $f_{c}=0.25,0.15$, and 0.05, respectively. As can be seen in Figure 16, a greater covering fraction leads to a softer spectrum, while an inhomogeneous corona with smaller yet denser clumps gives a larger optical depth for those photons that do not escape directly from the disk, in turn giving a larger Compton $y$-parameter and harder spectrum.

As mentioned above, the disk absorption in the AGN models results in a softer spectrum than stellar-mass BHs with the same coronal parameters. This can be seen by comparing Figure 16 with the upper-left panels in Figures 3 (wedge geometry; $f_{c}=1$ ) and 9 (clumpy geometry; $f_{c}=0.05$ ). In both cases, the spectral index $\alpha$ is increased (i.e., softer) in the AGN case by about 0.5 . The effect of this absorption on the X-ray polarization can be seen in Figure 17, which plots the degree and angle of polarization as a function of energy for the same model parameters used in Figure 16. As expected, for the wedge geometry with $f_{c}=1$, the absorbing disk leads to a larger degree of polarization than the reflecting boundary condition used in stellar-mass BHs $(\sim 6-8 \%$ for AGN compared with the $\sim 4 \%$ 
shown in Fig. 3 for $M=10 M_{\odot}$ and $\left.i=45^{\circ}\right)$.

For the clumpy coronae, however, we find the opposite effect: including absorption actually reduces the degree of polarization. Recall from Section 4 that inhomogeneous coronae generally produce a weaker polarization signal than the uniform-density sandwich geometry. This is because photons scattering multiple times in a single spherical cloud will ultimately escape with no net polarization. It is only through the global scattering geometry that a net vertical polarization is acquired, due to the small number of photons that scatter from one cloud to another, often reflecting off the disk surface at grazing incidence along the way. Since many of these photons are lost to absorption in the AGN case, global geometric effects play a smaller role, and thus the degree of polarization is diminished $(\sim 1 \%$ for AGN versus $\sim 2 \%$ for the stellar-mass case in Fig. 9). Thus we find that for AGN, X-ray polarization is even more sensitive to the inhomogeneity of the corona than for stellar-mass BHs.

From Figure 16, we see that it should be possible to determine the AGN covering fraction from the X-ray spectrum alone, without polarization information. If so, then the degree of polarization could rather be used to constrain the inclination of the AGN disk, a parameter that can be quite difficult to constrain in many cases. In Figure 18 we compare the degree of polarization for a range of inclination angles and two different corona models: wedge geometry with $f_{c}=1$ (solid curves) and a clumpy geometry with $f_{c}=0.15$ (dashed curves). Clearly the inclination will be easier to measure for a smooth corona, but even for a clumpy corona with $f_{c}=0.15$, a first-generation X-ray polarimeter should be able to distinguish between $i=15^{\circ}$ and $45^{\circ}$.

In the event that we can measure the covering fraction and inclination with other observations, the polarization can constrain other corona parameters like the number of clumps $n_{c}$ and their overdensity $\rho_{c} / \rho_{0}$. From equation (11), we see that for a constant covering fraction, $n_{c} \propto\left(\rho_{c} / \rho_{0}\right)^{2}$, so increasing the number of clouds both decreases their characteristic size and increases their density. Yet when averaged over a photon's entire path, a corona with larger $n_{c}$ will appear more homogeneous, despite the increased density perturbations on very small scales. This in turn leads to a greater degree of polarization, as shown in Figure 19. Holding the inclination and covering fraction fixed at $i=45^{\circ}$ and $f_{c}=0.15$, respectively, we vary the number density of coronal clumps. While all four cases plotted in Figure 19 have nearly identical spectra, we see that polarization can distinguish them and thus give improved constraints on the corona geometry. The different models in Figure 19 would also likely have observably different timing properties: if individual clumps evolve coherently, but are independent of one another, systems with smaller $n_{c}$ should produce greater amplitude flux variations in the X-ray band.

In practice, AGN polarization measurements will have some additional observational 
challenges not present in stellar-mass BH systems. First, the typical X-ray flux from nearby Seyfert galaxies and quasars is at least an magnitude smaller than the brightest galactic BHs. This means that much longer observation times will be required to reach a comparable level of polarization sensitivity. In addition, type 1 AGN are expected to have only modest inclinations $\left(i \lesssim 45^{\circ}\right)$ because at higher inclinations our view of the inner disk is likely blocked by the surrounding dusty torus (assuming the torus and disk are oriented in the same direction). There are also two potential sources of dilution. Radio-loud AGN generically have somewhat larger ratios of X-ray flux to optical flux than radio-quiet Shen et al. (2006), a fact plausibly interpreted as due to a jet contribution; in blazars, the jet contribution is almost certainly substantial. In addition, Fe K $\alpha$ emission often accounts for a few percent of the $2-10 \mathrm{keV}$ flux. Lastly, the much longer variability timescales for supermassive BHs make it unlikely that we could use the strategy discussed in Section 3, in which observations of multiple spectral states can jointly determine the orientation of the spin on the sky. This advantage would be absent in AGN systems, which for the most part do not undergo significant state transitions on an observable timescale.

\section{DISCUSSION}

Using a Monte Carlo ray-tracing code in the Kerr metric, we have explored a variety of models for X-ray-emitting coronae attached to accreting stellar-mass black holes in the hard/SPL states or to radio-quiet AGN. Over a wide range of model parameters, the polarization swings from parallel to the disk plane ("horizontal") at low energy to perpendicular ("vertical") at high energy. The location of this transition is generically at an energy a few times that of the highest temperature found in the disk; in the case of stellar-mass BHs, that means $\sim 1--3 \mathrm{keV}$; in $\mathrm{AGN}, \sim 100 \mathrm{eV}$. The detailed properties of this transition, and the polarization amplitude at the low- and high-energy limits, provide the observer with information about the BH's mass, spin, inclination, and accretion rate, as well as the coronal properties: degree of (in)homogeneity, vertical scale height, temperature, optical depth, and covering fraction.

We do not claim that a single or even multiple polarization observations of a given source will unambiguously measure all these model parameters. What does seem possible, even with relatively low precision data, is to rule out large regions of parameter space, and in so doing challenge a number of the traditional paradigms and toy models for $\mathrm{BH}$ accretion geometry. For example, if we were to observe an upper limit of $\delta \leq 2 \%$ at $\sim 10 \mathrm{keV}$ from a stellar-mass system with known binary inclination $i \gtrsim 60^{\circ}$, we would have only two choices: to abandon any form of smooth corona geometry in favor of a highly asymmetric, 
inhomogeneous accretion flow, or to posit a misalignment between the binary orbit and the inner accretion disk (thus giving lower inclination and polarization than expected). For AGN sources, where the expected disk inclination is lower $\left(i \lesssim 45^{\circ}\right)$, a null polarization measurement would rule out a smooth sandwich corona for anything but a nearly face-on system.

As with any theoretical model based on a large number of parameters, we expect that certain physical properties of the $\mathrm{BH}$ system will be more easily constrained than others. For example, even if we assume prior knowledge of the observer inclination, BH mass and accretion rate, as well as the temperature, density, and geometry of the corona, Figure 5 suggests that, with a polarization sensitivity of $\delta \lesssim 1 \%$, one could only just distinguish between a Schwarzschild and extreme Kerr BH in the hard state. However, this also means that even if we have relatively poor constraints on the spin parameter (which is generally the case for most galactic BHs), we should still be able to measure other parameters robustly. By measuring the power-law slope of the spectrum around $10 \mathrm{keV}$, one could estimate the Compton $y$-parameter with reasonable accuracy, but still face a degeneracy between the corona temperature and optical depth. Again assuming a known inclination and corona geometry, we see from Figure 6 that this degeneracy could be broken with a polarization observation.

As another example, if the coronal properties are well-known (perhaps through more detailed spectral observations over a greater range of energies, which could allow an independent determination of the electron temperature), the polarization could be used to determine the disk inclination. While the inclination could in principle be measured directly from the low-energy polarization (Connors et al. 1980; Li et al. 2008), first-generation polarimeters are likely not going to be very sensitive below $\sim 1 \mathrm{keV}$, where in any case, magnetic turbulence in the disk may reduce the net polarization via Faraday rotation (Davis et al. 2009). Yet as we see from Figure 3, a single high-energy polarization measurement at $\sim 10 \mathrm{keV}$ could give the inclination angle, assuming the other model parameters are known reasonably well. In practice, it is more likely that we could use some independent method (e.g. optical light curves, Fe line fitting, etc.) to determine the disk inclination, and then use the amplitude of polarization in the $\sim 1-10 \mathrm{keV}$ band to determine the inhomogeneity of the corona. Coronae with large-amplitude density fluctuations produce weaker polarization at high energies and a more gradual transition from horizontal to vertical orientation, as shown in Figures 10 and 11.

Poutanen et al. (1997) showed that, using basic physical arguments about the covering fraction of the corona and the relative flux in the hard and soft components, the smooth sandwich corona could be distinguished from a patchy corona on the basis of the spectral 
properties alone. Yet in Section 5, we have shown that the spectrum from a truncated disk around a spherical corona can be nearly indistinguishable from that of a wedge corona above an extended disk. However, the polarization signatures from these two models are quite distinguishable: the spherical geometry leads to a horizontal-vertical transition at lower energy and gives significantly weaker polarization at all energies.

As seen in Sections 3 and 4, the sense of rotation in the polarization transition (i.e., $\psi>0$ or $\psi<0$ ) contains additional information that constrains the corona scale height and level of inhomogeneity. The angle of polarization at low energies defines the projection of the disk plane on the sky, but not the direction of the BH spin (as in many binary systems, the line of nodes is uniquely determined, but it is impossible to know which is the ascending node). From observations of the thermal state, we can use the sense of the rotation toward vertical polarization to determine the sense of orbital motion of the disk, thus defining the $\psi>0$ direction. Given that result, the sign of $\psi$ in the coronal state can constrain the scale height of a wedge corona (Fig. 7) or the covering fraction of a clumpy corona (Figs. 10 and 11).

Additional constraints on clumping properties $\rho_{c} / \rho_{0}$ and $n_{c}$ can come from the amplitude of polarization above the thermal peak as follows: In stellar-mass systems, we might determine the inclination of the disk from radial velocity observations of the companion. In AGN, the inclination may be inferred from the blue edge of a broad iron line (Reynolds \& Nowak 2003). In either stellar-mass systems or AGN, the slope of the hard power-law tail of the X-ray spectrum can give the covering fraction $f_{c}$, which is related to $\rho_{c} / \rho_{0}$ and $n_{c}$ through equation (11). For a given $i$ and $f_{c}$, the degree of polarization at high energies can then be used to determine the coronal properties $\rho_{c} / \rho_{0}$ and $n_{c}$.

Furthermore, with timing observations we expect an inverse correlation between the amplitude of hard X-ray luminosity fluctuations and the number of coronal clumps, due simply to Poisson statistics. In a related way, polarization could provide valuable insight into the nature of poorly-understood timing phenomena such as quasi-periodic oscillations (QPOs). The QPOs observed in stellar-mass BHs are most prominent in the hard X-rays $(\geq 6 \mathrm{keV})$, even in the SPL state when most of the flux is at lower energies (Remillard \& McClintock 2006), suggesting that the QPOs come from the hot corona. By comparing the polarization signal with the timing properties at different epochs, we should be able to constrain the location, size, and coherence of the regions from which QPOs originate. For example, if we find that periods of large amplitude timing fluctuations correlate with low-amplitude polarization, then it is quite likely that both features are caused by a relatively small number of hot, dense clouds orbiting above the inner disk.

Lastly, in Section 6, we showed how the special properties of AGN could be exploited 
through X-ray spectropolarimetry. Because the seed photons in such systems are at much lower energies, more scattering events are required to inverse Compton scatter the seed photons to X-ray energies. Additionally, because the relatively cool disk is largely opaque to absorption below $\sim 10 \mathrm{keV}$, the range of available scattering angles is more restricted. Together, these features particular to AGN systems lead to a stronger ability to discriminate between different coronal covering fractions, as well as the characteristic size and density of the scattering clouds. Combining spectral and polarization information, it should be possible to determine the coronal covering fraction and the disk inclination independently with moderate precision. In contrast to the galactic BHs, in the AGN case it is unlikely that we will be able to observe a single system in multiple accretion states, yet the much longer time scales should allow for more detailed comparisons between polarization and variability.

We would like to thank Tim Kallman, Jean Swank, and Shane Davis for helpful discussions and comments. This work was supported by the Chandra Postdoctoral Fellowship Program grant PF7-80051 (JDS) and NSF grant AST-0908336 (JHK). 


\section{REFERENCES}

Agol, E., \& Krolik, J. H. 2000, ApJ, 528, 161

Bardeen, J. M., Press, W. H., \& Teukolsky, S. A. 1972, ApJ 178, 347

Bellazzini, R., et al. 2006, Nucl. Instr. Meth., A560, 425

Black, J. K., et al. 2003, Nucl. Instr. Meth., 513, 639

Blandford, R. D., \& Begelman, M. C. 2004, MNRAS, 349, 68

Carter, B. 1968, Phys. Rev., 174, 1559

Chandrasekhar, S. 1960. Radiative Transfer, Dover, New York

Connors, P. A., \& Stark, R. F. 1980, Nature, 269, 128

Connors, P. A., Piran, T., \& Stark, R. F. 1980, ApJ, 235, 224

Costa, E., et al. 2008, Proc. SPIE, vol. 7011-15, arXiv:0810.2700

Cunningham, C. T. 1976, ApJ, 208, 534

Davis, S. W., Blaes, O. M., Hirose, S., Krolik, J. H. 2009, ApJ 703, 569

Dolence, J. C., Gammie, C. F., Moscibrodzka, M., Leung, P. K. 2009, [arXiv:09090708]

Done, C., \& Zycki, P. T. 1999, MNRAS, 305, 457

Done, C., \& Trigo, M. D. 2009, MNRAS submitted, [arXiv:0911:3243]

Dovciak, M., Karas, V., \& Matt, G. 2004, MNRAS, 355, 1005

Dovciak, M., Muleri, F., Goodmann, R. W., Karas, V., \& Matt, G. 2008, MNRAS submitted, arXiv:0809.0418

Elvis, M., et al. 1978, MNRAS, 183, 129

Esin, A. A., et al. 2001, ApJ, 555, 483

Gierliński, M., et al. 1997, MNRAS, 288, 958

Gierliński, M., \& Done, C. 2005, MNRAS, 347, 885

Haardt, F., \& Maraschi, L. 1993, ApJ, 413, 507 
Haardt, F., Maraschi, L., \& Ghisellini, G. 1994, ApJ, 432, L95

Ingram, A., Done, C., \& Fragile, P. C. 2009, MNRAS, 397, L101

Jahoda, K., Black, K., Deines-Jones, P., Hill, J. E., Kallman, T., Strohmayer, T., \& Swank, J. 2007, [arXiv:0701090]

Laor, A., Netzer, H., \& Piran, T. 1990, MNRAS, 242, 560

Li, L.-X., Narayan, R., \& McClintock, J. E. 2008, ApJ submitted, arXiv:0809.0866

Makishima, K., et al. 2008, PASJ, 60, 585

Matt, G., Fabian, A. C., \& Ross, R. R. 1993, MNRAS, 264, 839

McClintock, J. E., et al. 2001, ApJ, 555, 477

Miller, J. M., et al. 2006, ApJ, 653, 525

Mushotzky, R. F., Done, C., \& Pounds, K. A. 1993, ARA\& A, 31, 717

Nandra, K., et al. 1991, MNRAS, 248, 760

Noble, S. C., Krolik, J. H., \& Hawley, J. F. 2008, ApJ submitted, arXiv:0808.3140]

Novikov, I. D., \& Thorne, K. S. 1973, in Black Holes, ed. C. DeWitt \& B. S. DeWitt (New York: Gordon and Breach)

Pietrini, P. \& Krolik, J.H. 1995, ApJ, 447, 526

Poutanen, J., Krolik, J. H., \& Ryde, F. 1997, MNRAS, 292, L21

Poutanen, J., \& Fabian, A. C. 1999, MNRAS, 306, L31

Reis, R. C., Fabian, A. C., \& Miller, J. M. 2009, MNRAS accepted, arXiv:0911.1151]

Remillard, R. A., \& McClintock, J. E. 2006, ARA\& A, 44, 49

Reynolds, C. S., \& Nowak, M. A. 2003, Phys. Reports, 377, 389

Rybicki, G. B., \& Lightman, A. P. 1979, Radiative Processes in Astrophysics (New York: Wiley-Interscience)

Schnittman, J. D., \& Krolik, J. H. 2009, ApJ 701, 1175

Schnittman, J. D., \& Krolik, J. H. 2010, in preparation 
Shakura, N. I., \& Sunyaev, R. A. 1973, A\&A, 24, 337

Shen, Shiyin, White, S.D.M., Mo, H.J., Voges, W., Kauffmann, G., Tremonti, C. \& Anderson, S.F. 2006, MNRAS 369, 1639

Shimura, T., \& Takahara, F. 1995, ApJ, 445, 780

Stark, R. F., \& Connors, P. A. 1977, Nature, 266, 429

Stern, B. E., Poutanen, J., Svensson, R., Sikora, M., \& Begelman, M. C. 1995, ApJ, 449, L13

Sunyaev, R. A., \& Titarchuk, L. G. 1985, A\&A, 143, 374

Swank, J., et al., in X-ray Polarimetry: A New Window in Astrophysics, Rome 2009, edited by R. Bellazzini, E. Costa, G. Matt and G. Tagliaferri

Titarchuk, L., \& Shrader, C. 2002, ApJ, 567, 1057

Turolla, R., Zane, S., \& Titarchuk, L. 2002, ApJ, 576, 349

Walker, M., \& Penrose, R. 1970, Commun. Math. Phys., 18, 265

Zdziarski, A. A., \& Gierliński, M. 2004, Prog. Theor. Phys. Suppl., 144, 99

Zdziarski, A. A., Gierliński, M., Rao, A. R., Vadawale, S. V., \& Mikalojewska, J. 2005, MNRAS, 360, 825 
Fig. 1.- Schematic diagram of the disk and corona for the sandwich geometry. Thermal seeds are emitted from a thin disk in the midplane, then scatter off hot electrons in a corona with a wedge geometry and constant scale height $H / R$. The optical depth to electron scattering in the vertical is constant throughout the disk. For observers at high inclination (edge-on), the scattered photons will likely have high polarization, oriented perpendicular to the scattering plane, as indicated by the small black lines. Some photons emitted from the inner disk are deflected by the $\mathrm{BH}$ and then scatter off the disk on the far side, also leading to large amplitude polarization.

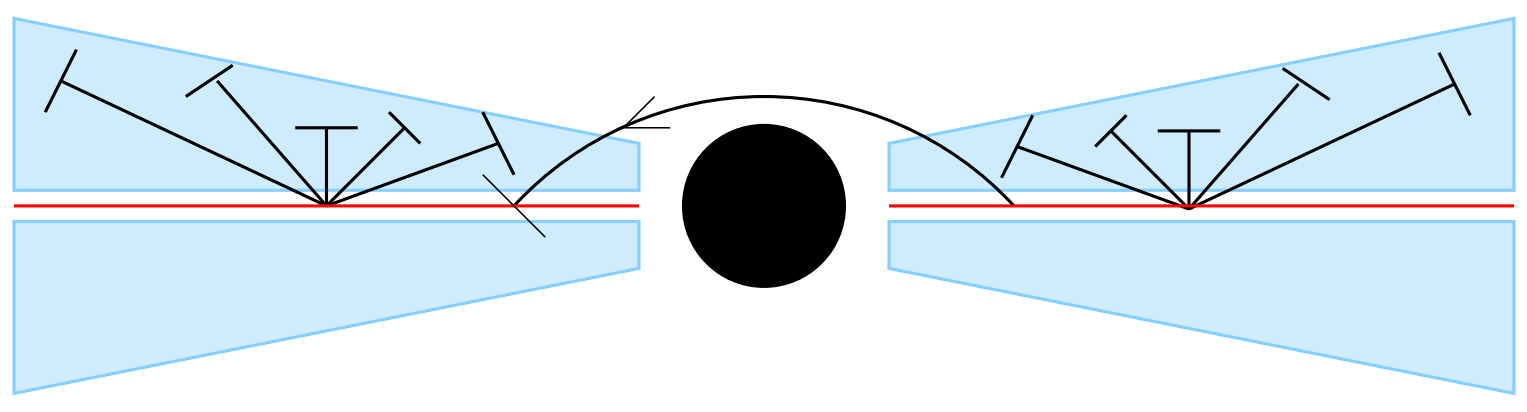


Fig. 2.- Ray-traced images of polarized flux from an accretion disk with a sandwich corona of scale height $H / R=0.1$. The observer is located at an inclination of $75^{\circ}$ relative to the rotation axis, with the gas on the left side of the disk moving towards the observer, which causes the characteristic increase in intensity due to relativistic beaming and boosting. The black hole has spin $a / M=0.9$, mass $M=10 M_{\odot}$. The observed intensity is color-coded on a logarithmic scale (normalized to the net intensity $\int d \nu I_{\nu}$ ), and the energy-integrated polarization vectors are projected onto the image plane with lengths proportional to the local degree of polarization. The four panels correspond to the contributions to the observed flux (a) directly from the thermal disk; (b) return radiation scattered once off the disk; (c) photons scattered once in the corona; and (d) photons scattered multiple times in the corona.
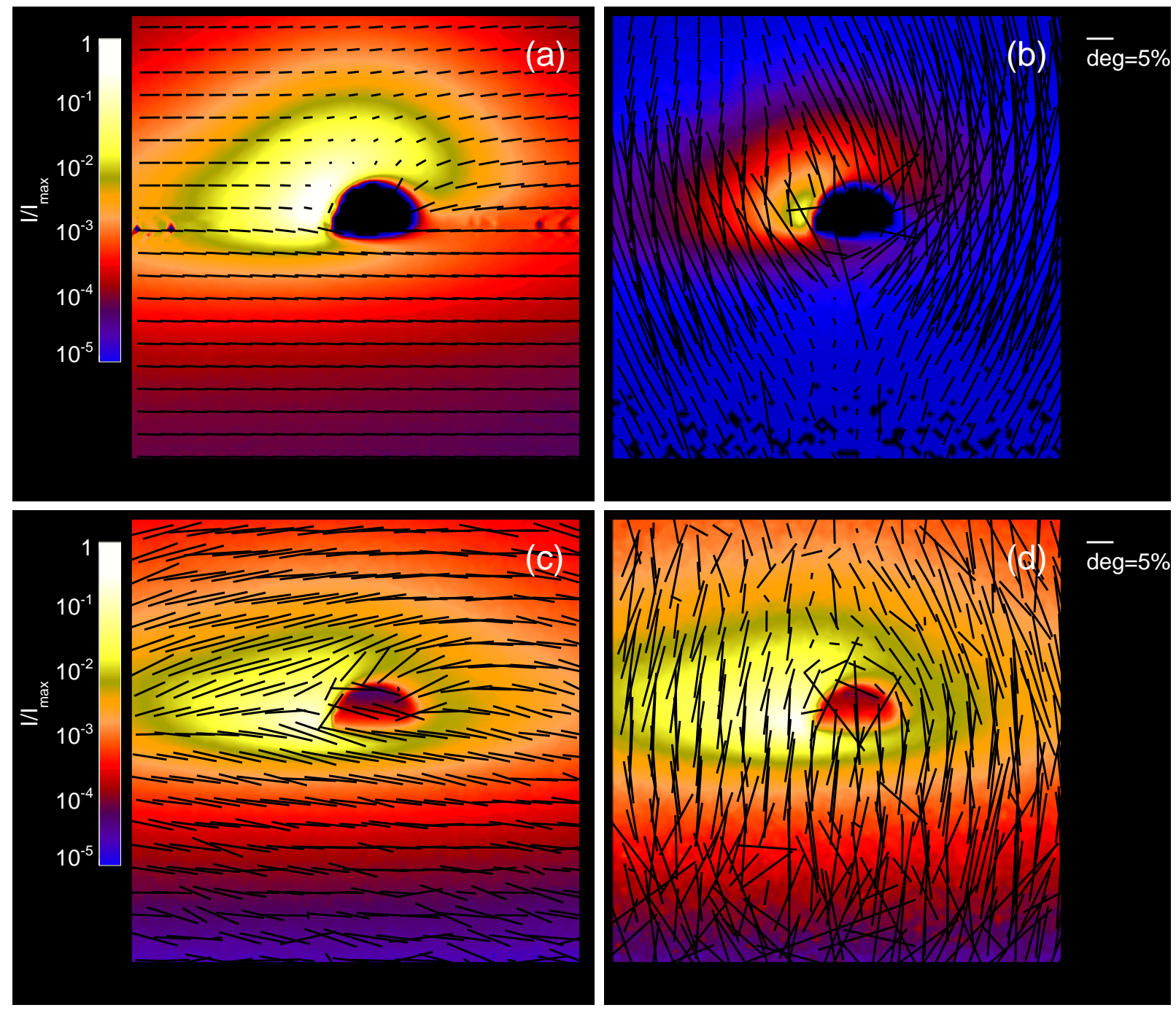
Fig. 3.- Observed flux and polarization from an accretion disk with a sandwich corona geometry. The plots show the flux (left; arbitrary units of $\nu F_{\nu}$ ), polarization degree (center), and polarization angle (right) as a function of observed energy, for inclinations of $45^{\circ}, 60^{\circ}$, and $75^{\circ}$ (top, center, bottom, respectively). The dotted lines represent contributions directly from the thermal disk, the dot-dashed curves are corona-scattered photons, and the solid curves are the total observed flux.
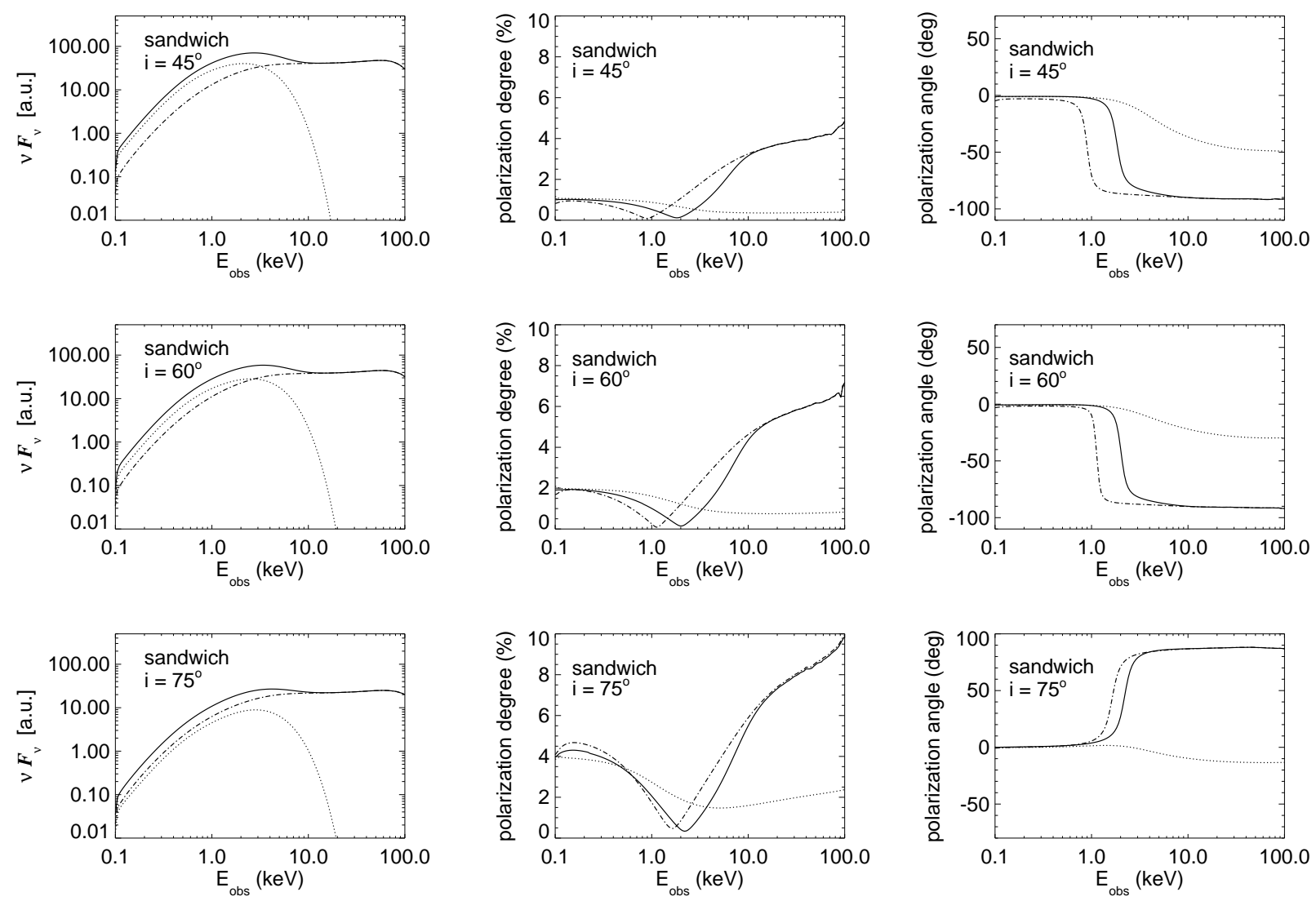
Fig. 4.- Degree and angle of polarization for a sandwich corona, varying the luminosity in the thermal flux.
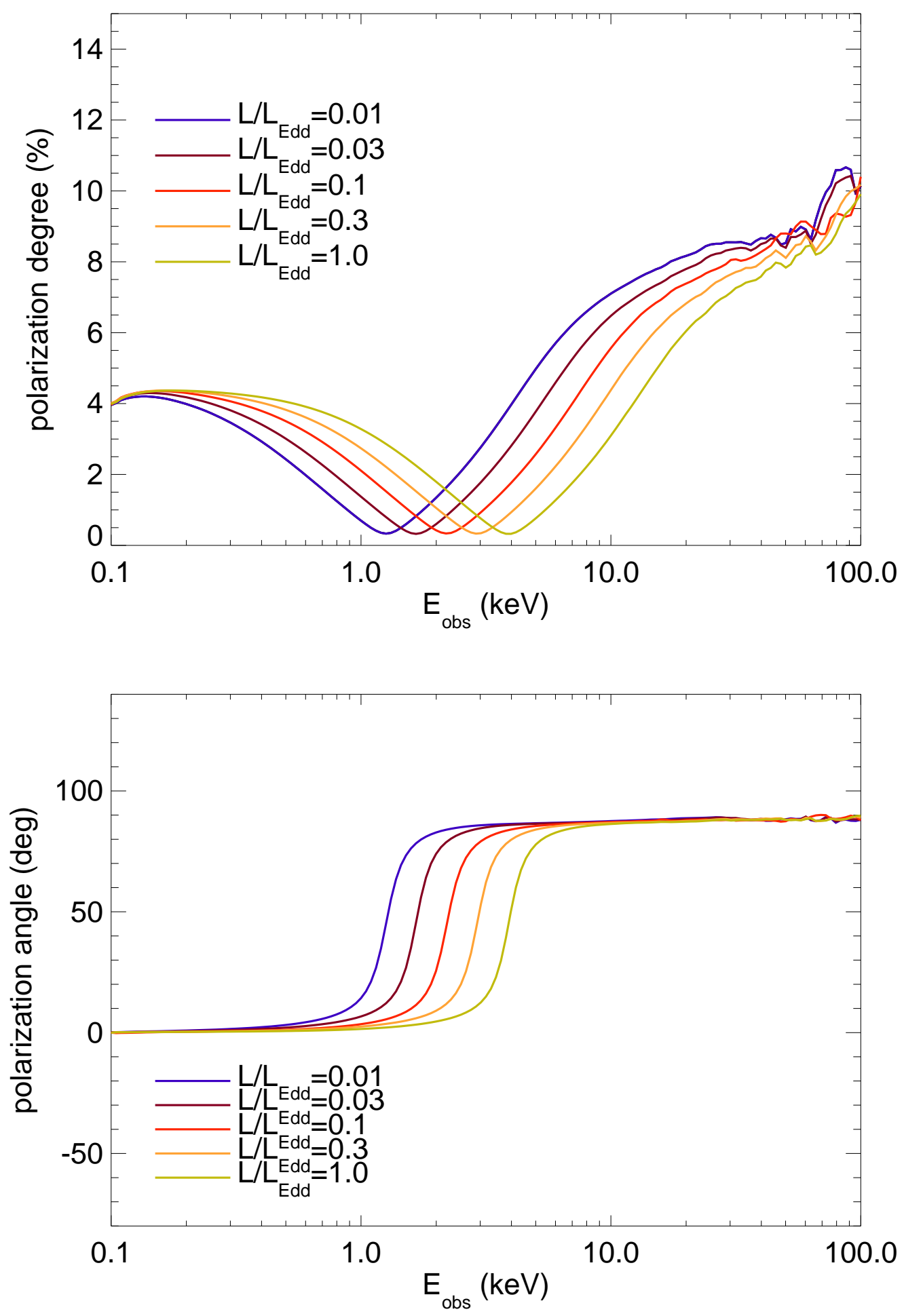
Fig. 5.- Degree and angle of polarization for a sandwich corona, varying the spin of the $\mathrm{BH}$, while holding fixed the total thermal flux.
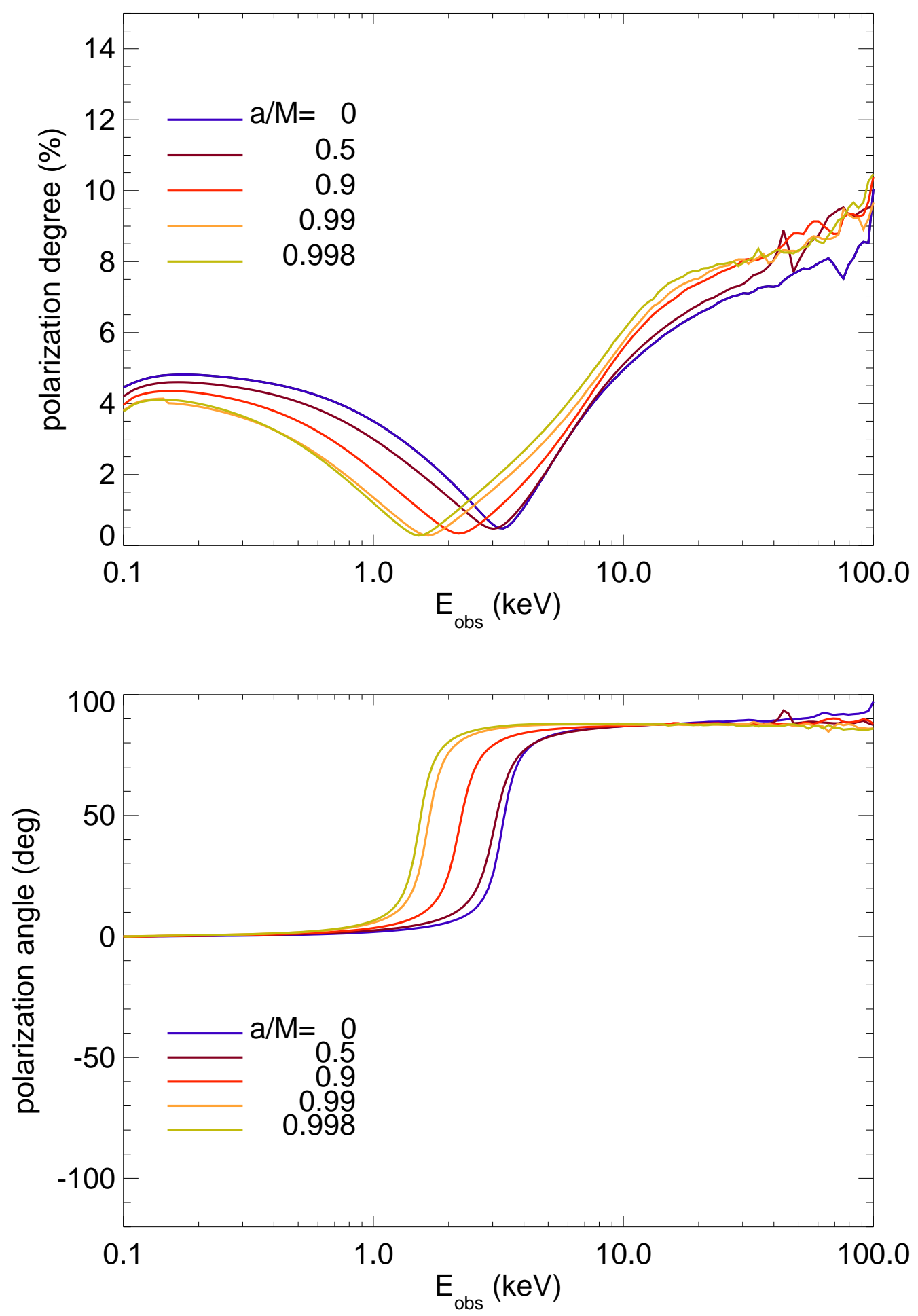
Fig. 6.- Degree and angle of polarization for a sandwich corona, varying the optical depth and electron temperature, maintaining a roughly constant Compton- $y$ parameter.
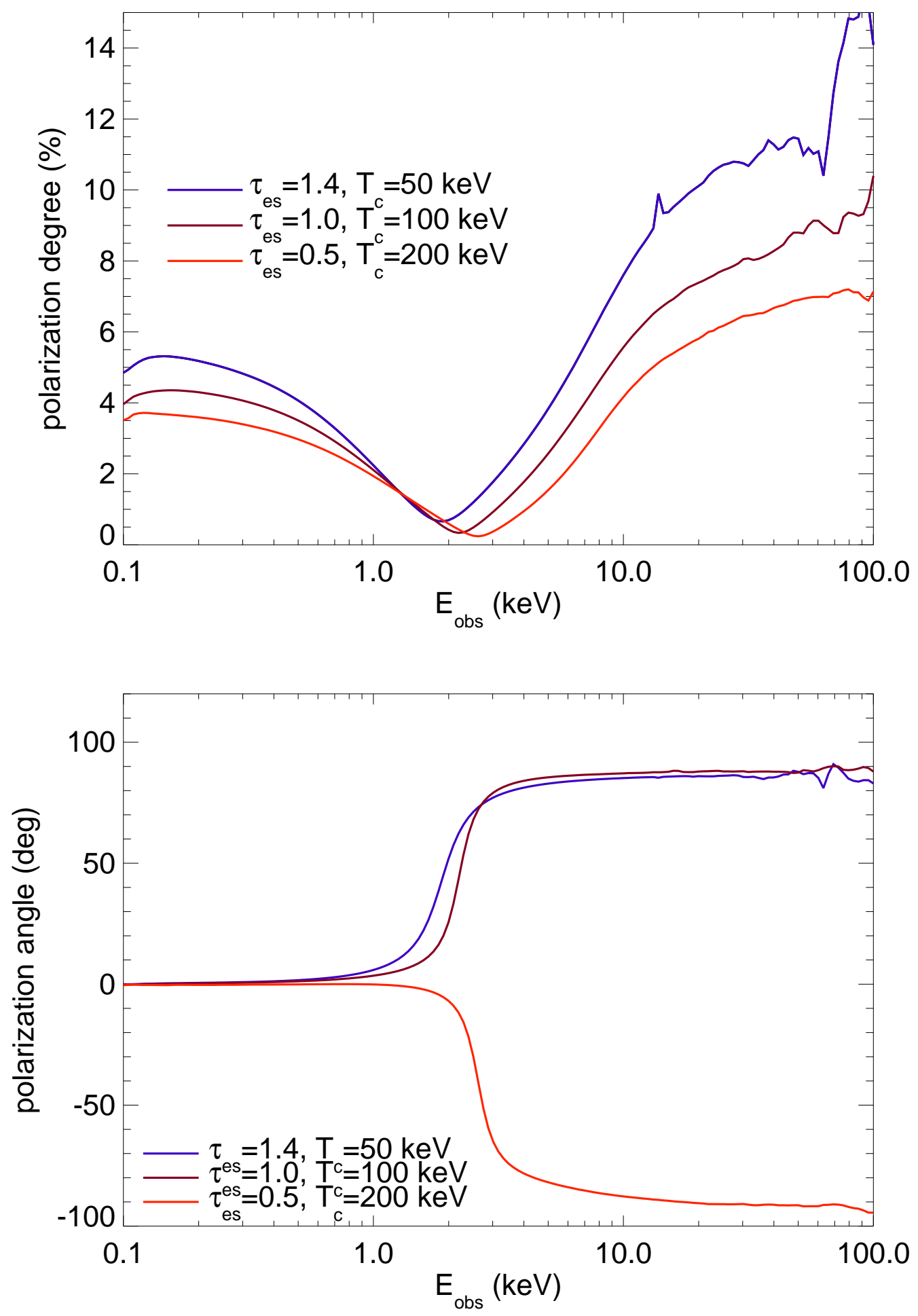
Fig. 7.- Degree and angle of polarization for a sandwich corona, varying the scale height of the corona.
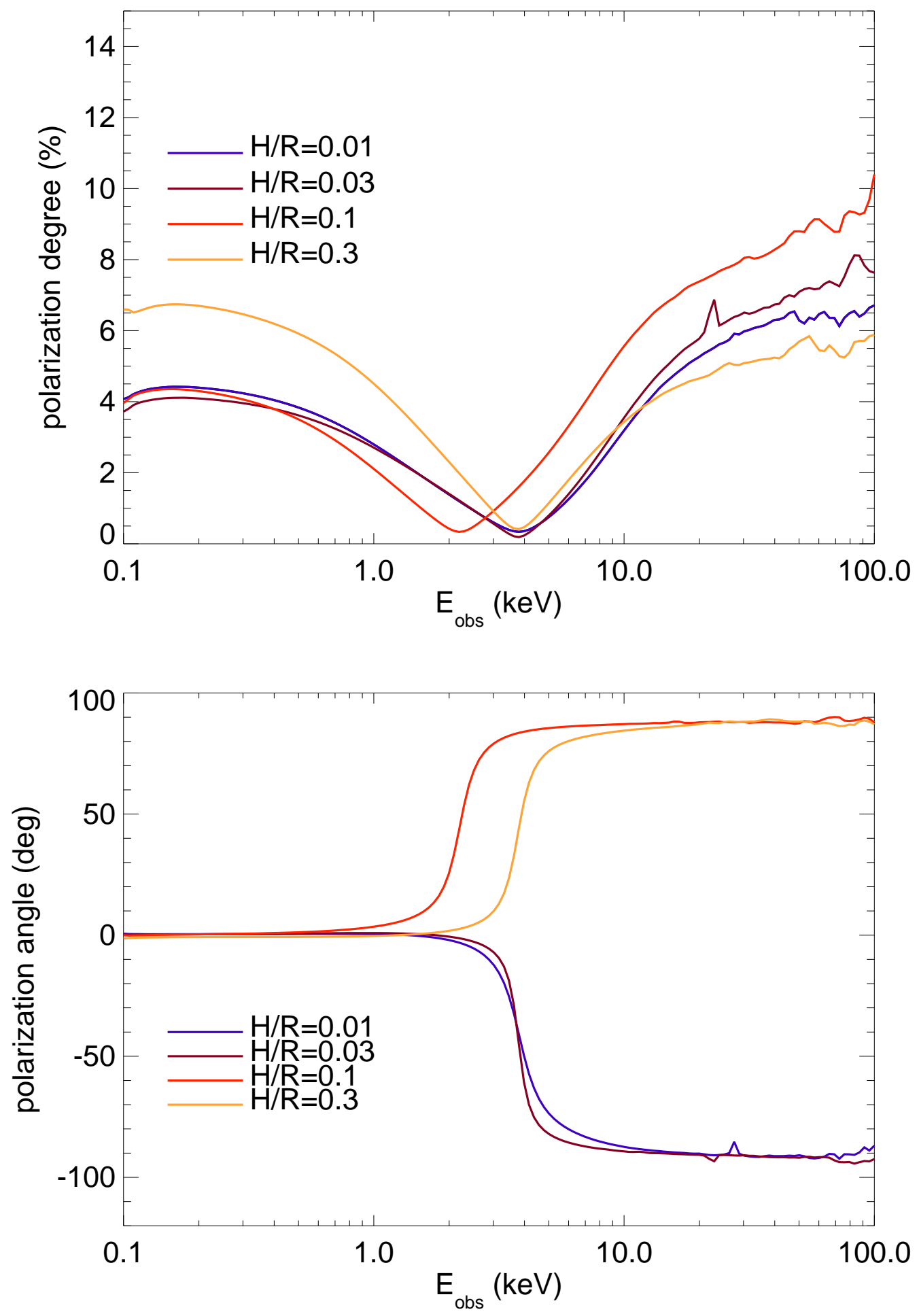
Fig. 8. - Same as Figure 2, but for a hot spot corona with an overdensity factor of 10 . There are roughly 100 hot spots distributed randomly within radius $R \leq 100 M$ with scale height $H / R=0.1$.
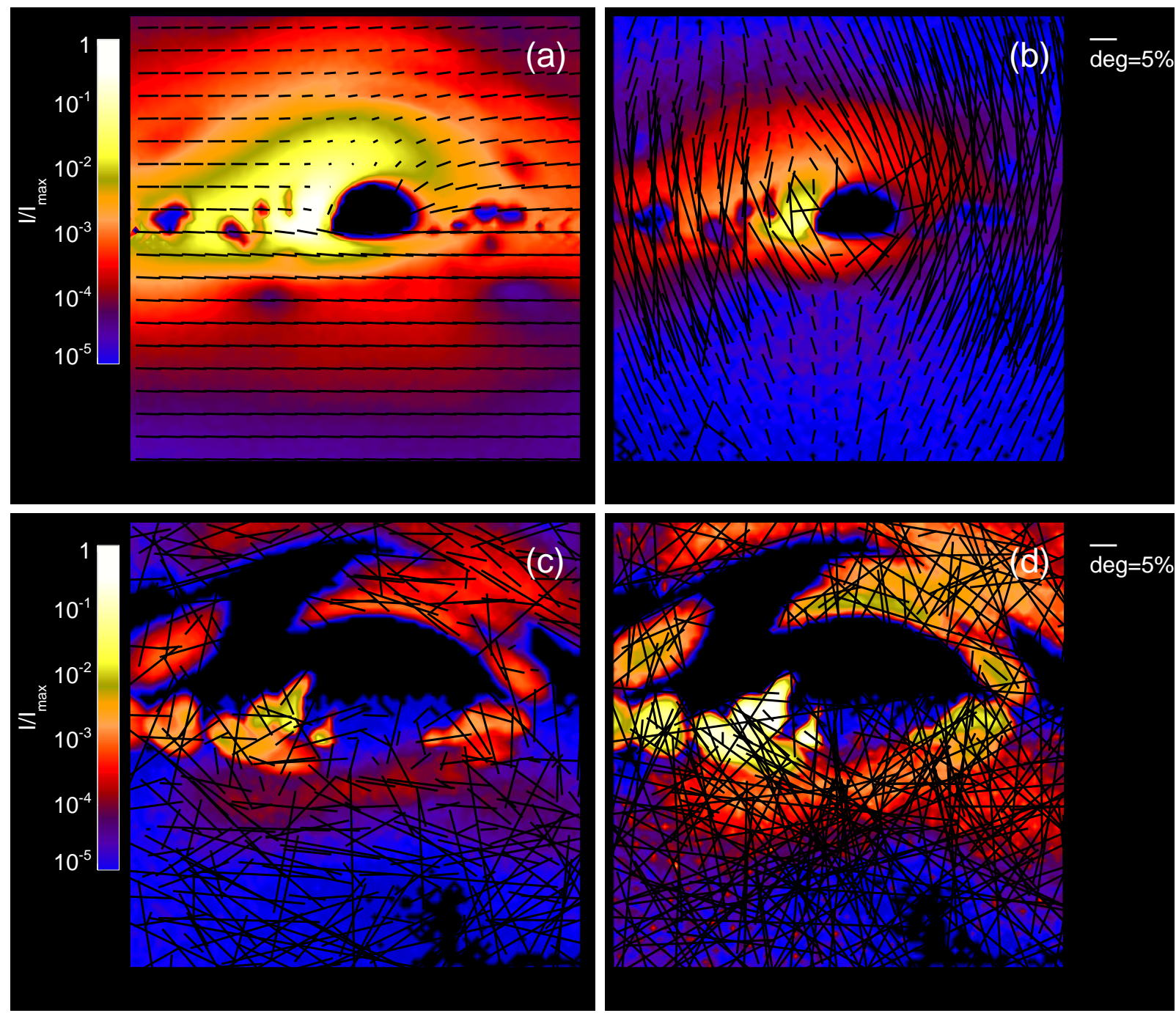
Fig. 9.- Same as Figure 3, but for the hot spot corona geometry shown in Figure 8
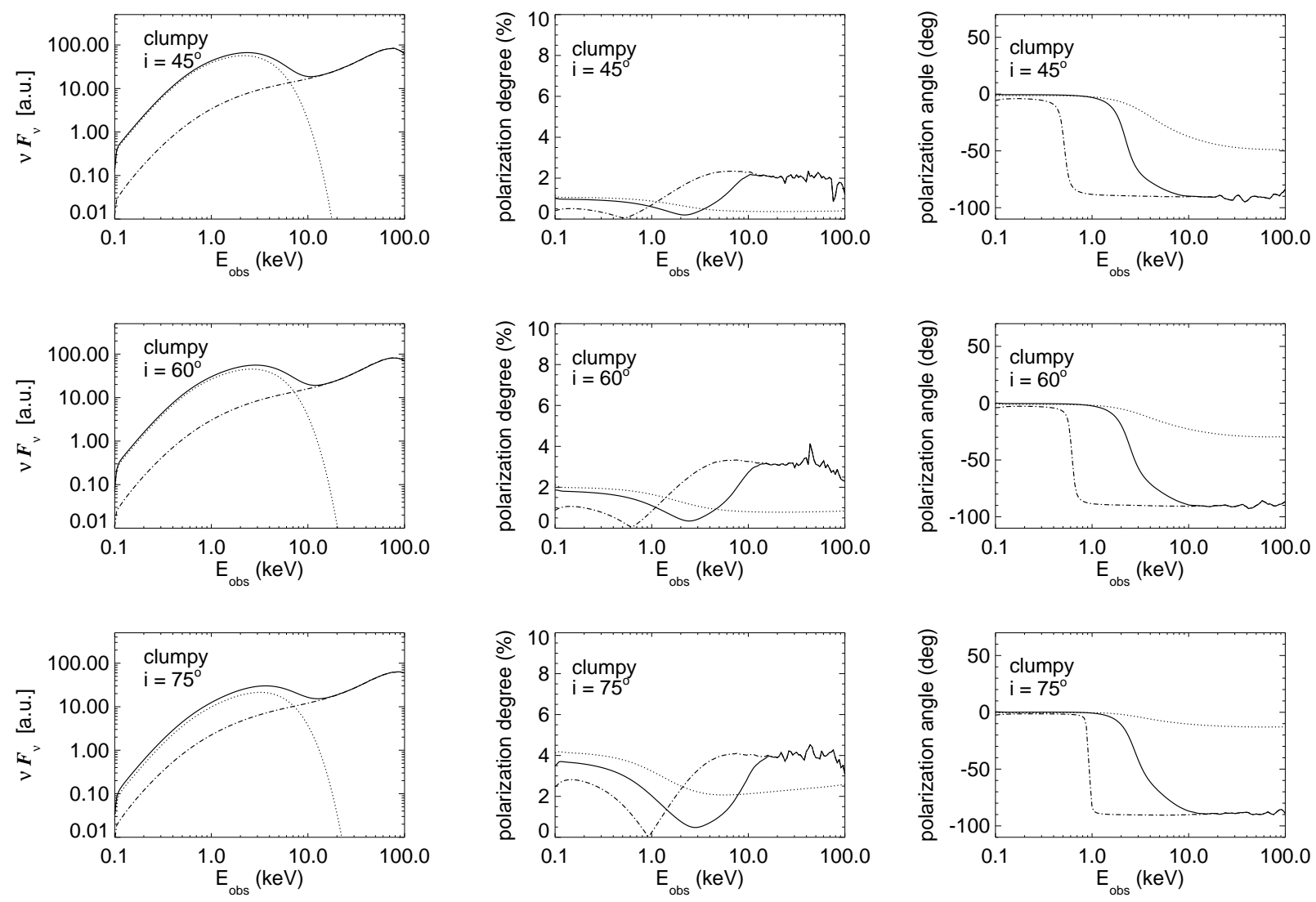
Fig. 10.- Degree and angle of polarization for a hot spot corona, varying the compactness of the coronal hot spots.
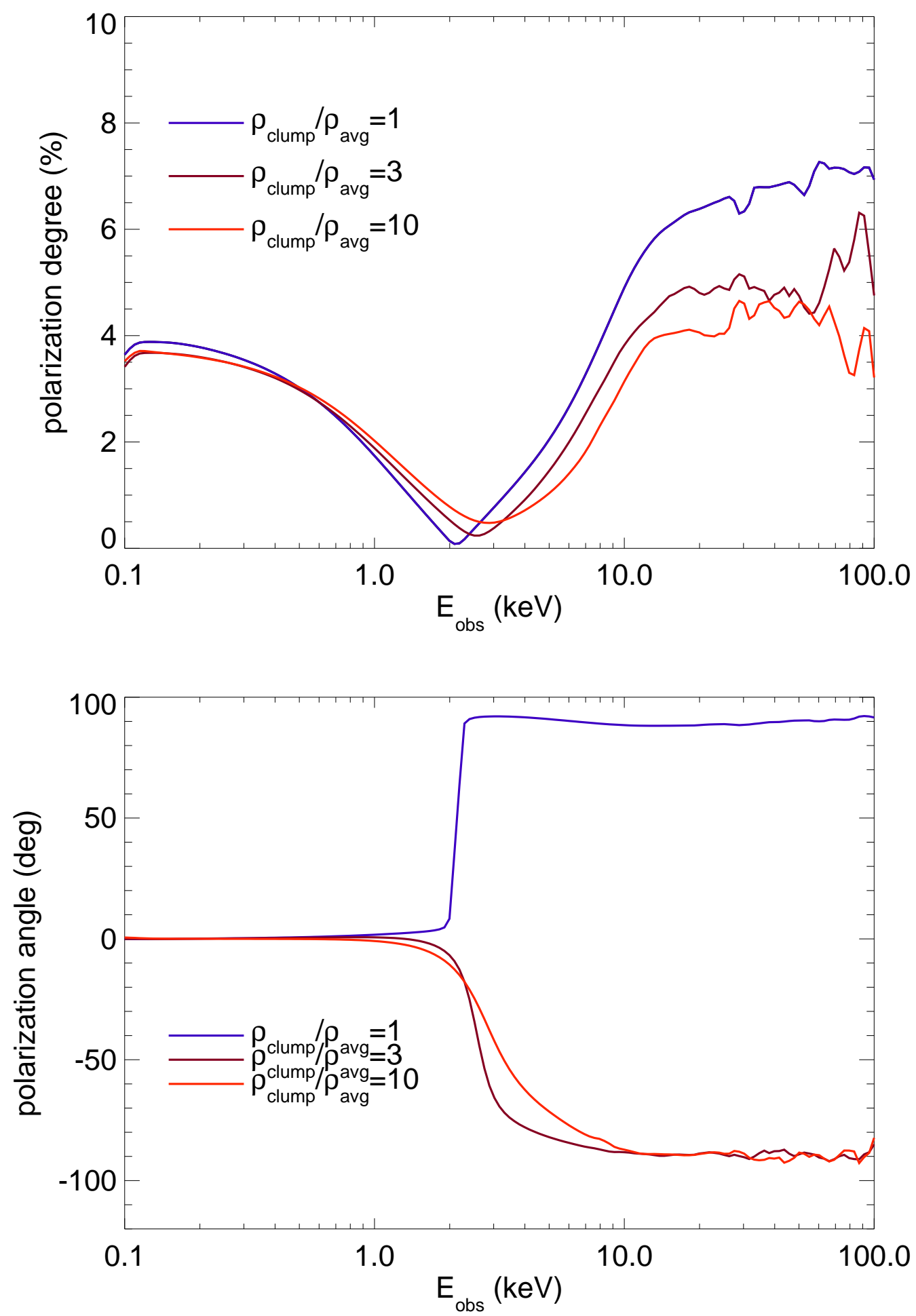
Fig. 11. - Degree and angle of polarization for a hot spot corona, varying the number density of coronal hot spots.
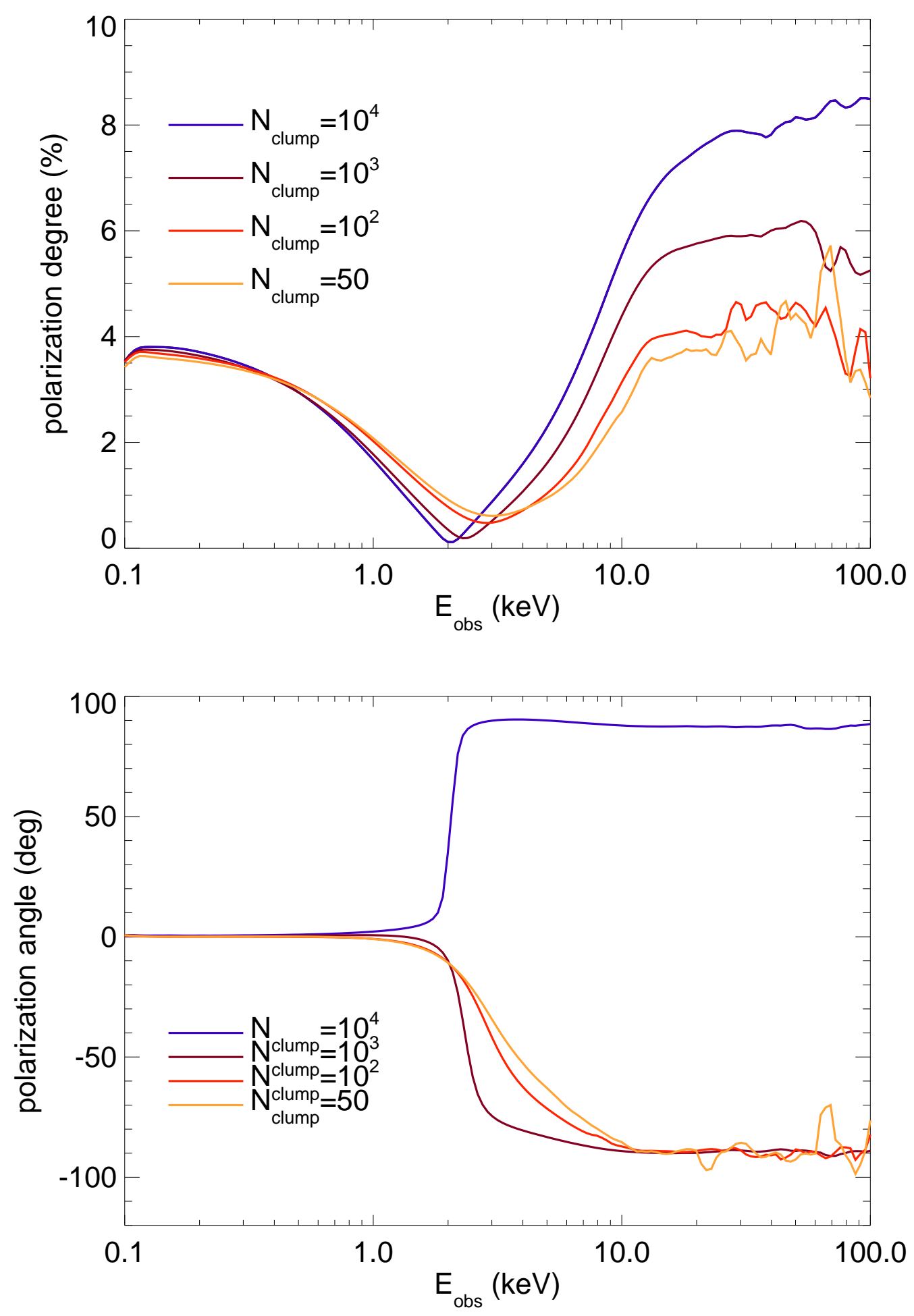
Fig. 12.- Same as Figure 2, but for a spherical corona surrounded by a truncated thermal disk with an inner edge at $R_{\text {edge }}=15 \mathrm{M}$.
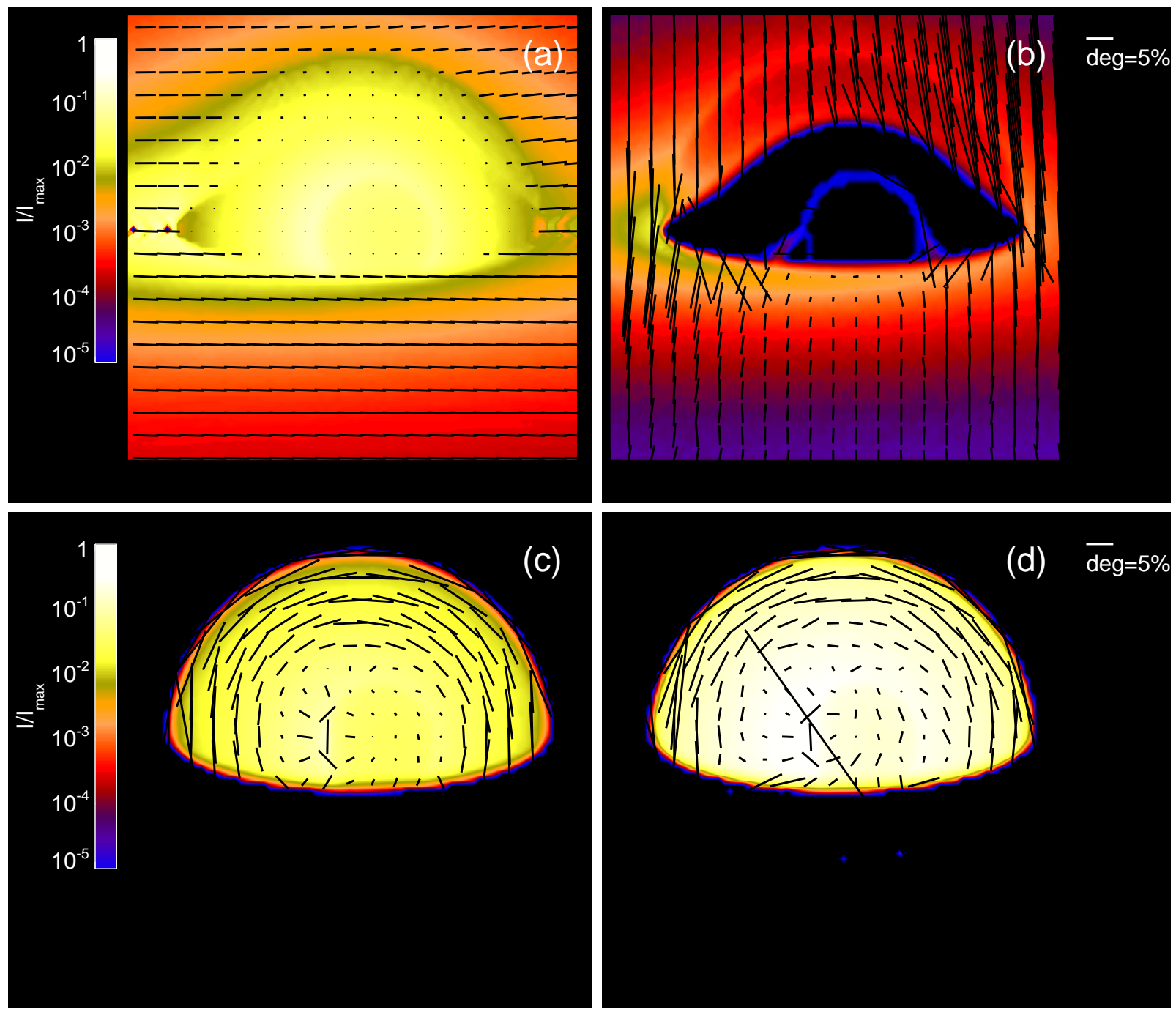
Fig. 13. - Same as Figure 3, but for the spherical corona geometry shown in Figure 12.
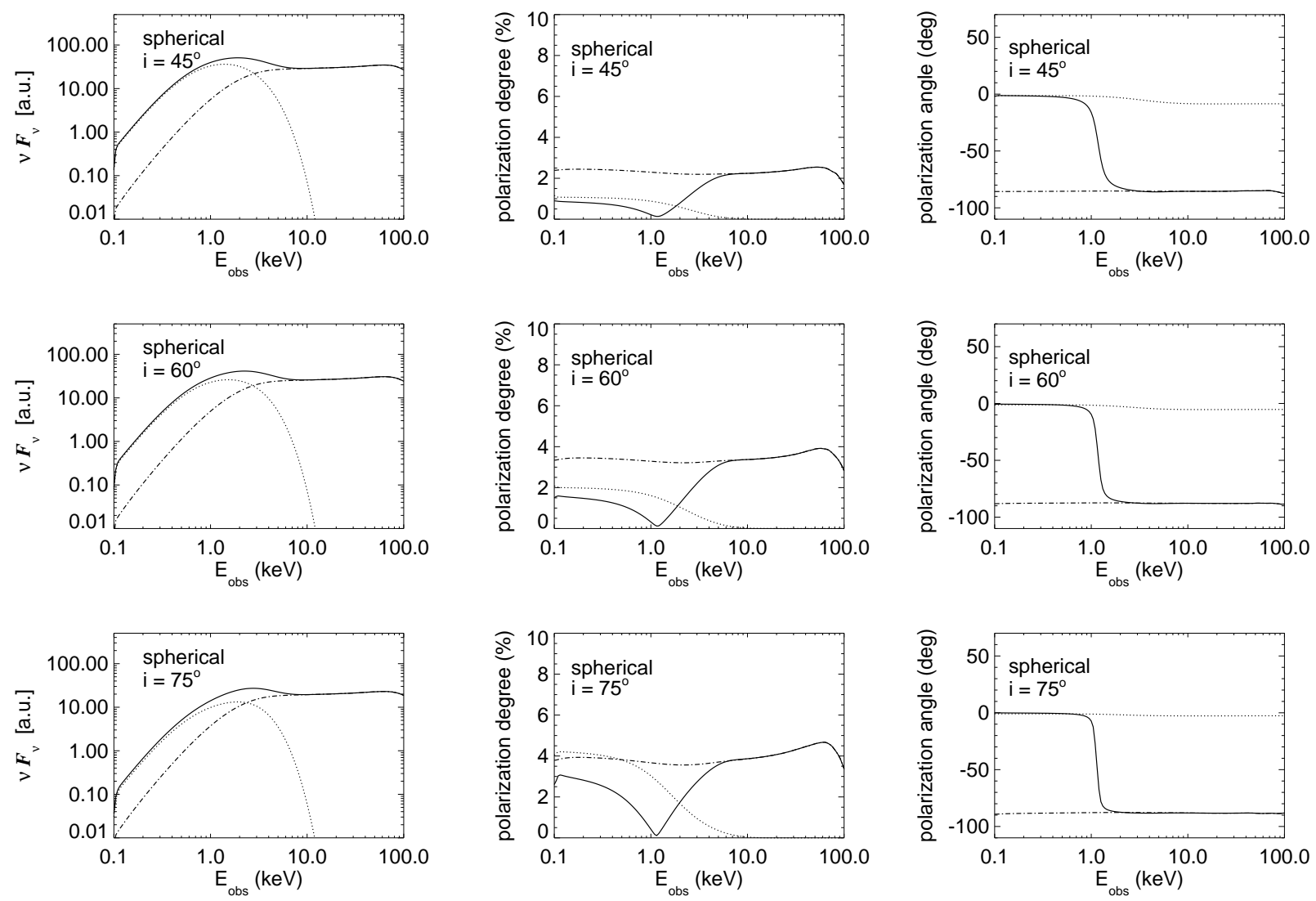
Fig. 14. - Degree and angle of polarization for a spherical corona, varying the radius of the corona (inner edge of the disk).
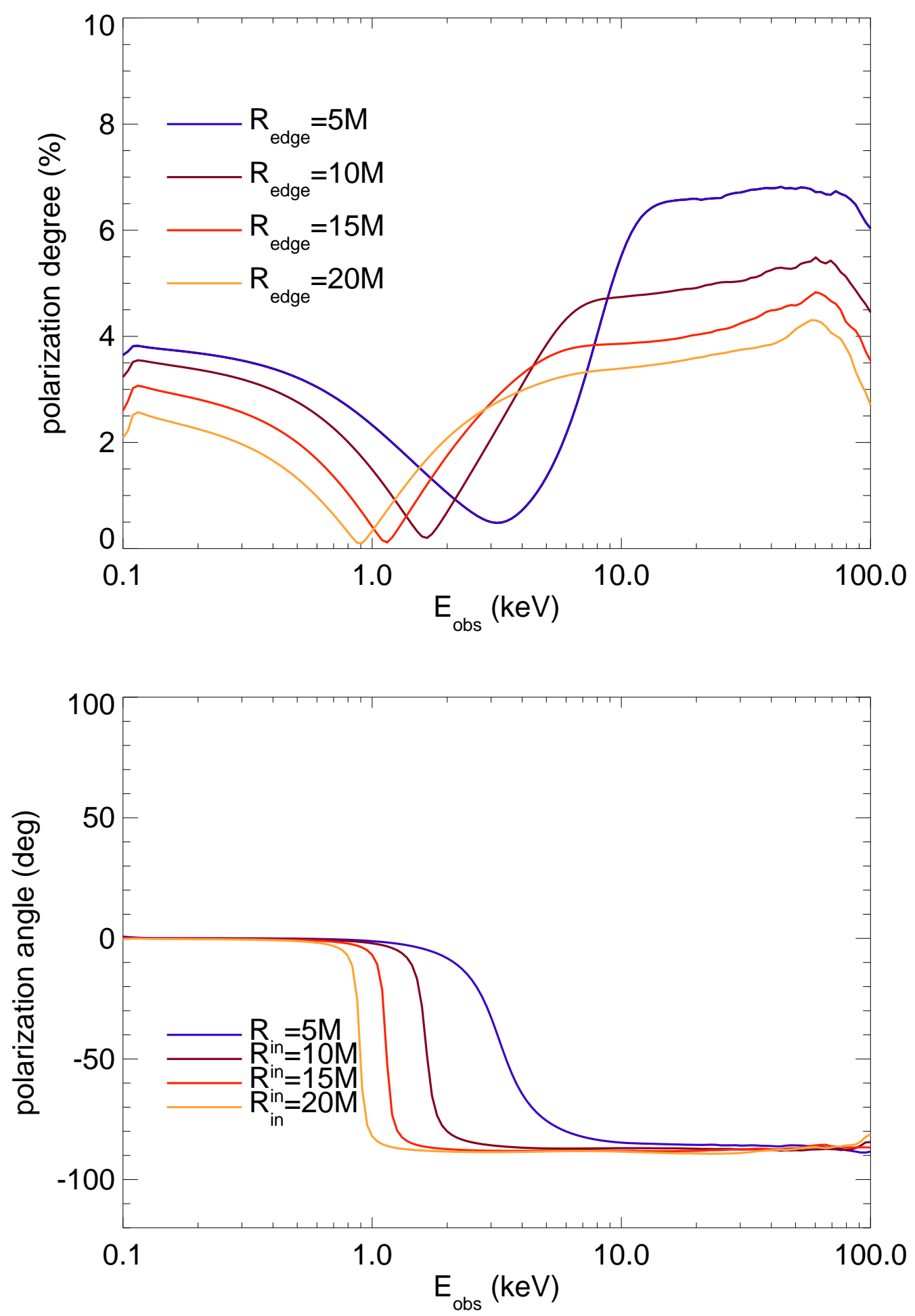
Fig. 15.- Degree and angle of polarization for a spherical corona, fixing $R_{\text {edge }}=10 M$ and varying the optical depth and electron temperature.
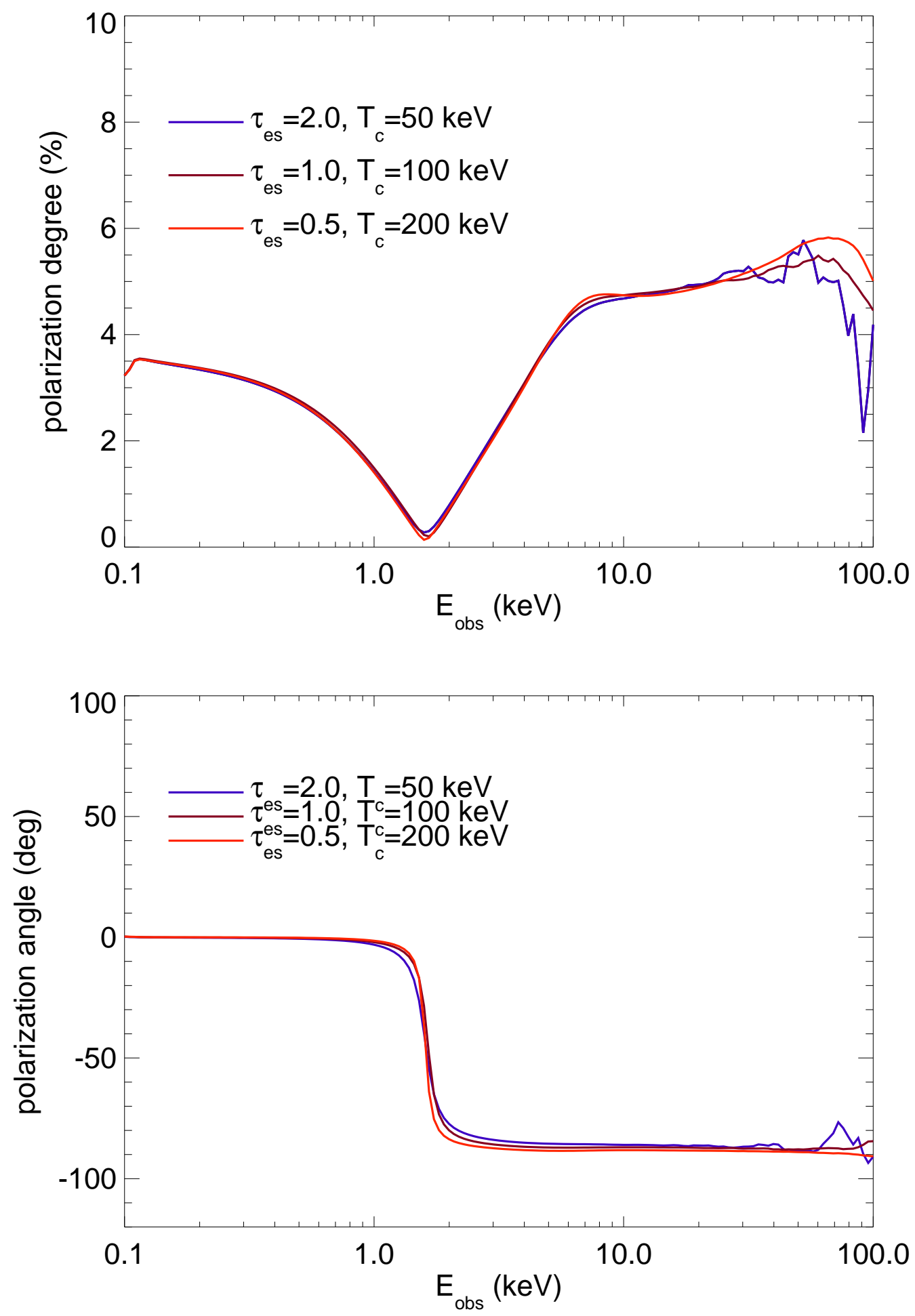
Fig. 16.- Observed broad-band flux from a supermassive BH with $M=10^{7} M_{\odot}, a / M=0.9$, $L_{\text {therm }}=0.1 L_{\mathrm{Edd}}$, and $i=45^{\circ}$. The different curves correspond to different covering fractions, as described in the text.

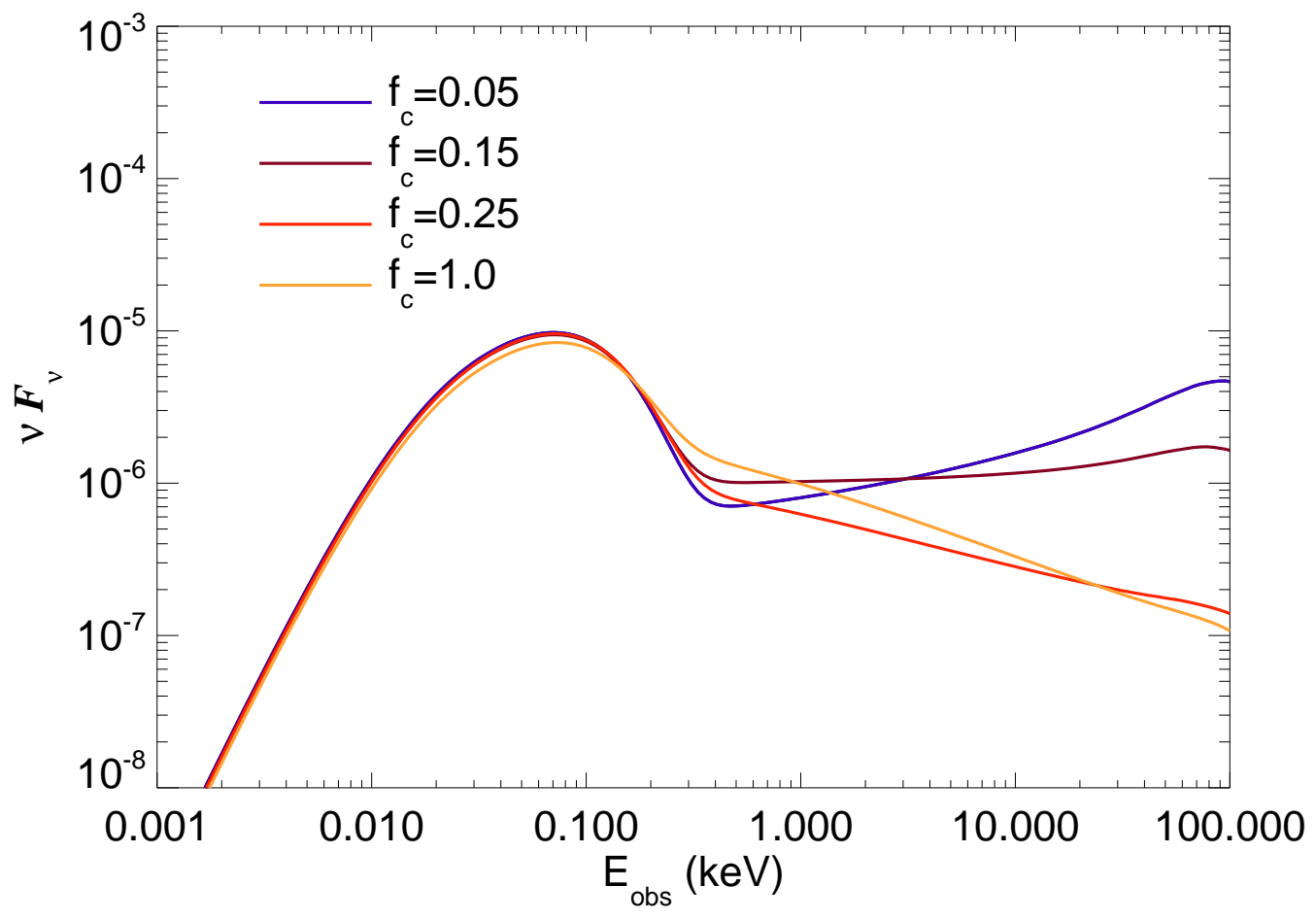


Fig. 17.- Degree and angle of polarization for a supermassive BH with the same parameters as in Figure 16.
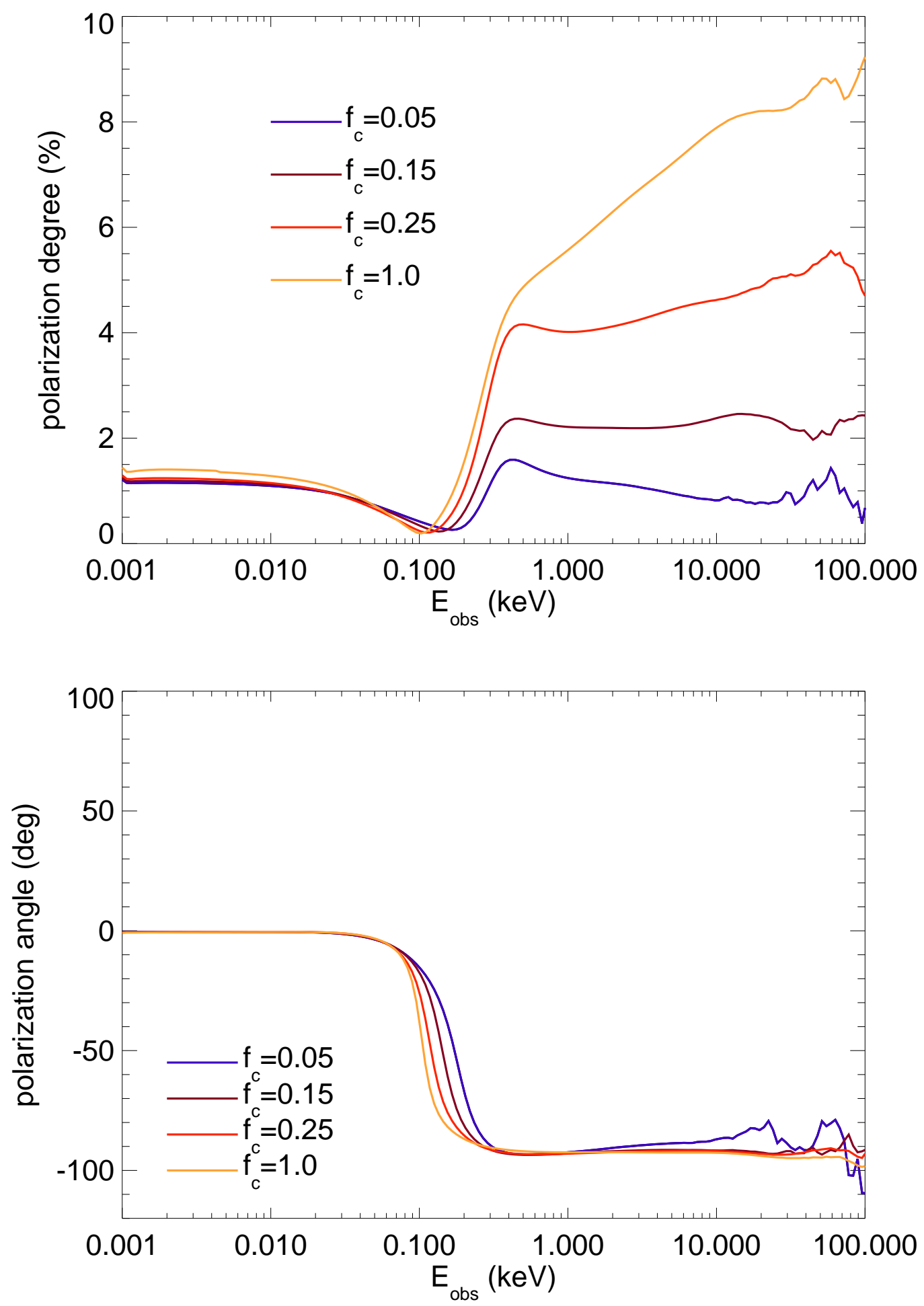
Fig. 18. - Degree of polarization for a supermassive BH with covering fractions $f_{c}=0.15$ (dashed curves) and $f_{c}=1$ (solid curves), for a range of observer inclination angles.

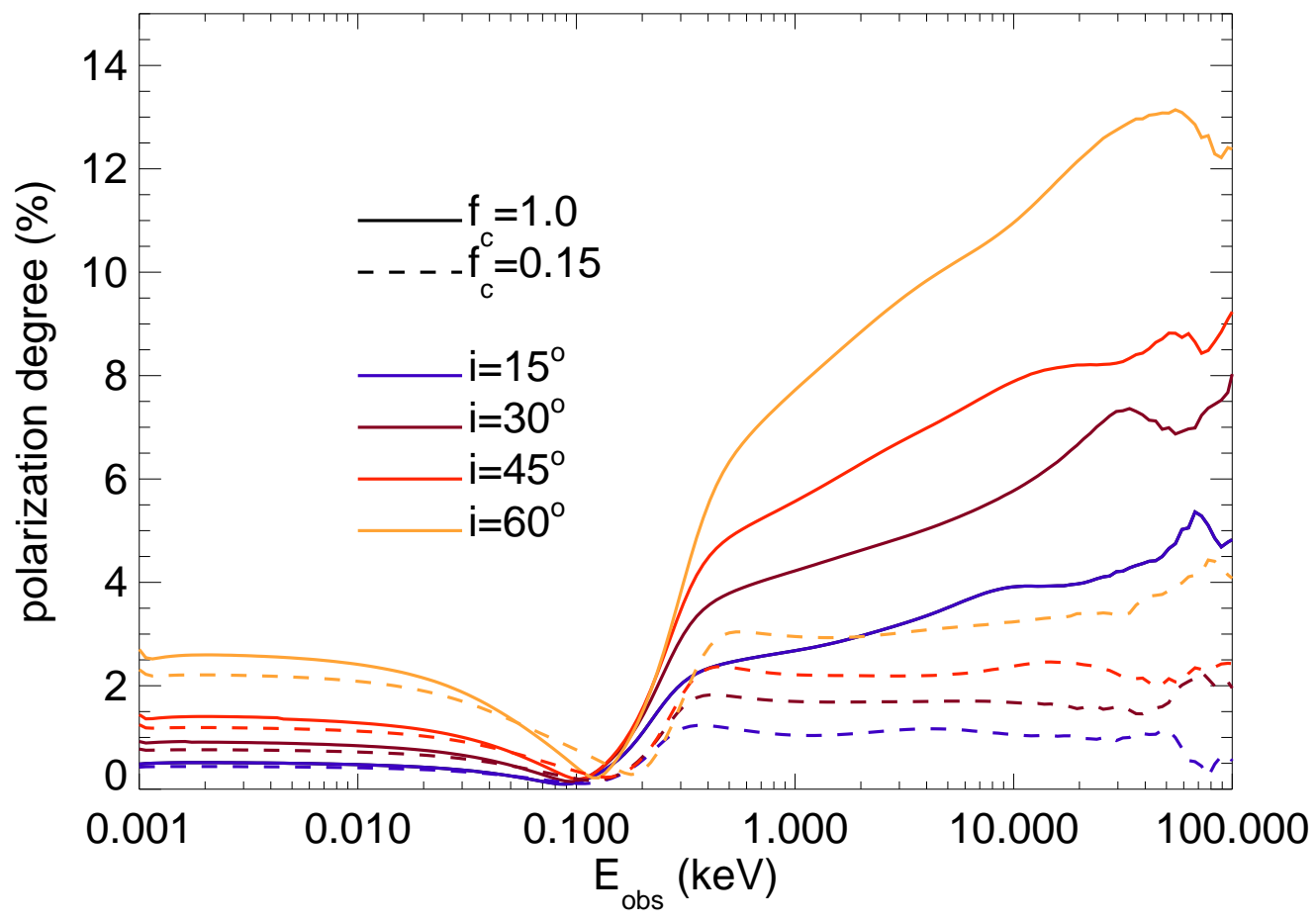


Fig. 19. - Degree of polarization for a supermassive $\mathrm{BH}$ with covering fraction $f_{c}=0.15$, inclination $i=45^{\circ}$, and a range of clump sizes. From equation (11) we see that, for a constant covering fraction, the number density of clouds $n_{c}$ is a function of the overdensity parameter $\rho_{c} / \rho_{0}$.

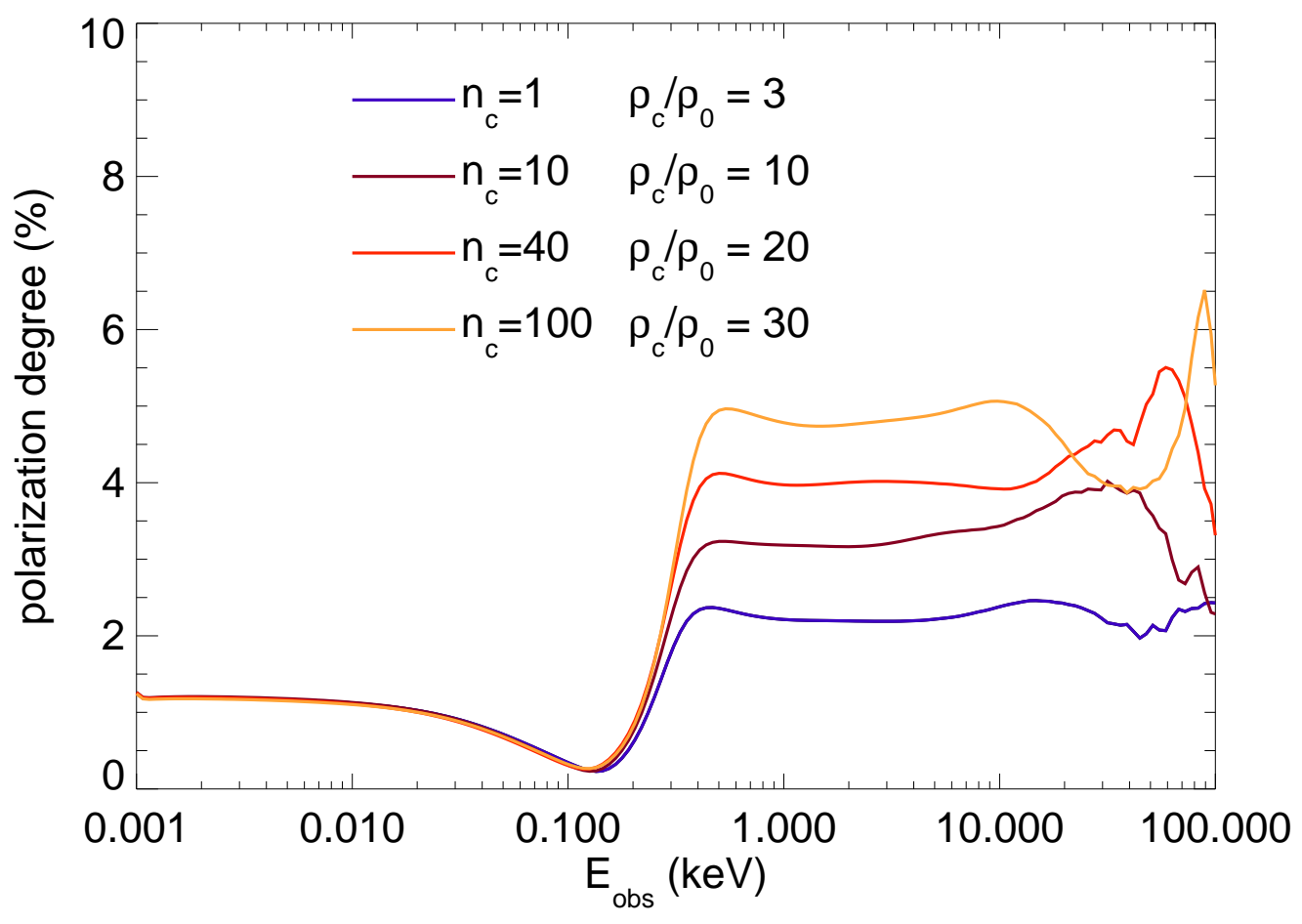

